Notre Dame Journal of Formal Logic

Volume XII, Number 3, July 1971

NDJFAM

\title{
WEAK FORMS OF THE AXIOM OF CONSTRUCTIBILITY
}

\author{
RICHARD L. POSS
}

TABLE OF CONTENTS

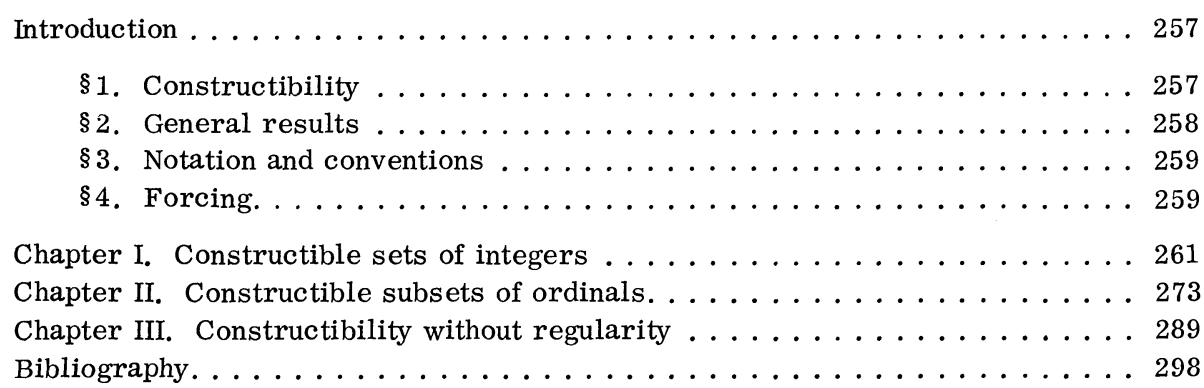

\section{INTRODUCTION*}

§1. Constructibility. The notion of constructibility in set theory was first mentioned by Kurt Gödel in [7], in the year 1938. Roughly speaking, he said that a set is "constructible" if it can be obtained from the empty set by the elementary set operations applied transfinitely many times. We then say that if every set in our theory is constructible the axiom of constructibility holds. Gödel used the axiom of constructibility to prove the consistency of the axiom of choice and of the generalized continuum hypothesis (under the assumption that set theory itself is consistent). To do this, he exhibited a model in which the axiom of constructibility holds and then showed that the axiom of constructibility implies the generalized continuum hypothesis and, hence, the axiom of choice.

*This work is based on a dissertation submitted in partial fulfillment of the requirements for the Ph.D. degree in Mathematics at the University of Notre Dame, August, 1970. The author wishes to express his gratitude to Prof. Bolesław Sobocinski for directing this research. 
In 1963, P. J. Cohen [2] showed that the axiom of constructibility was not provable in set theory and later [3] he gave a proof that it was not provable in set theory even if we assume the truth of the generalized continuum hypothesis. More recently, Gaifman [6], Rowbottom [14], Scott [17], Silver [21], and Solovay [22] have shown that the axiom of constructibility is incompatible with the existence of certain large cardinal numbers.

$\S 2$. General results. The purpose of this thesis is to investigate some of the ways in which the axiom of constructibility can be weakened. One recent method was that used by $B$. Scarpellini in [16]. He worked with the assumption that there is some set $m_{0}$ from which every set is constructible. In other words, starting with this $m_{0}$ we could, by use of the elementary set operations applied transfinitely many times, eventually "construct' every set in our universe.

Azriel Lévy, in [9], developed another weakening of the axiom of constructibility. He added another "elementary set operation" and considered the sets that now became constructible. By this method he proved some of the theorems about constructible sets which we will discuss in Chapters II and III.

Another approach was that of J. R. Shoenfield, in [19]. He employed the assumption that every set of integers is constructible in an attempt to further determine whether the axiom of constructibility is independent of set theory. We will study this assumption and determine some of its consequences by using the techniques of Cohen, which were unavailable at the time that Shoenfield published his paper.

The first chapter of this thesis consists entirely of considering this assumption that every set of integers is constructible. We will see that this is a consistent assumption (if set theory is consistent) and that it is not provable in set theory. We will then show that this assumption is actually weaker than the axiom of constructibility and that, in fact, assuming that all sets of integers are constructible is not sufficient to prove the axiom of choice.

In the second chapter we will generalize the results of the first chapter. There we will assume that all the subsets of some ordinal number are constructible. We will see that the consequences of this assumption are very closely related to the results of Chapter I. We will then study the relationships between these assumptions for various ordinal numbers. It happens that this method is helpful in distinguishing between regular and singular cardinal numbers. Finally, in this chapter, we give a result that in some ways extends the main theorem of Easton's thesis [5]. The general theme of this chapter is that assuming that all the subsets of a certain ordinal number are constructible does not necessarily affect the properties of subsets of higher ordinal numbers.

The third chapter differs considerably from the first two. There we will study the axiom of constructibility in a theory which does not have the axiom of regularity. We will then show that the axiom of constructibility can be properly decomposed into the axiom of regularity plus a proposition which states that if every element of a set is constructible then that set is constructible. 
§3. Notations and Conventions. In most of our discussions we will be working in Zermelo-Fraenkel set theory. The axiomatization that we shall use will be that of Cohen [3], pp. 50-53; namely, the axioms of extensionality, null set, unordered pairs, union, infinity, replacement, power set, and regularity. At times it may prove advisable to use a different axiomatization or a different set theory, but in these cases we will state specifically in what system we are working. We shall use the following abbreviations: $\mathrm{AC}$ for axiom of choice, $\mathrm{CH}$ for continuum hypothesis, and $\mathrm{GCH}$ for generalized continuum hypothesis. If we wish to add a proposition $S$ as an axiom to $Z F$, we will denote the new theory by $\mathbf{Z F}+\mathbf{S}$.

We assume famir:arity with Gödel [8], and, following his notation and definitions, use $\mathrm{V}(x), \operatorname{On}(x), \mathrm{L}(x)$, and $\mathrm{Od}(x)$ to mean, respectively, that " $x$ is a set," " $x$ is an ordinal number," " $x$ is constructible," and "the order of $x$." An expression of the form " $x \in \mathrm{L}$ ", will be interpreted to mean $\mathrm{L}(x)$; hence, we can use the standard notation $V=L$ as an abbreviation for the axiom of constructibility.

We shall use standard notation wherever possible. Small Greek letters, $\alpha, \beta, \ldots$ will denote ordinal numbers. Small Latin letters will represent sets, except that letters $p$ and $q$ will usually be reserved for forcing conditions. Capital letters will usually represent formulas. $\boldsymbol{p}(x)$ will always mean "the power set of $x$." Hence, the formula $\boldsymbol{P}(x) \subset \mathrm{L}$ will mean: "every subset of $x$ is constructible." We shall use $\phi$ as the empty set. We shall use $x \cap y$ for " $x$ intersect $y$ "; $x \cup y$ for "the union of $x$ and $y$ "; $\bigcup x$ for the set of all $z$ such that there is a $y$ such that $z \epsilon y$ and $y \in x ; \mathfrak{A} \rightarrow \mathfrak{B}$ for " $\mathscr{A}$ implies $\mathfrak{B}$ "; $\mathfrak{A} \leftrightarrow \mathscr{B}$ for " $\mathfrak{A}$ is equivalent to $\mathfrak{B}$ "; $\mathfrak{A} \wedge \mathfrak{B}$ for " $\mathscr{A}$ and $\mathfrak{B}$ "; $\mathfrak{A} \vee \mathfrak{B}$ for " $\mathfrak{A}$ or $\mathfrak{B}$ "; $\sim \mathfrak{A}$ for "not $\mathfrak{U}$ "; $\forall x \mathfrak{U}$ for "for all $x \mathfrak{U}$ "; $\exists x \mathfrak{U}$ for "there is an $x$ such that $\mathscr{A}$." $x \times y$ for the Cartesian product of $x$ and $y$. If $x$ is a set, then $\overline{\bar{x}}$ will be the cardinality of $x$. We shall say that a set $x$ is transitive if, for every $y \epsilon x, z \epsilon y$ implies $z \epsilon x$. Other special terms will be defined as we need them.

$\S 4$. Forcing. We assume some familiarity with the notion of forcing and the results of Cohen [3]. Here, however, we shall use forcing as modified by Lévy and Solovay in [10] and [11]. We will now present a brief description of forcing as presented in [11]. Rather than present it in the full generality, we will merely give the version that we will need for the results of Chapters I and II.

Even though we have nothing in ZF that we can properly call classes, we shall use the term in an intuitive manner to mean a subcollection of the universe of sets of the form $\{x \mid \mathfrak{A}(x, t)\}$. We shall use the term "subclass" in a similar manner. Here $\mathfrak{A}$ is a formula of ZF with two free variables and the set $t$ serves as parameter. By a "Cohen extension" we mean the following. We are given a set $C$, whose members we call conditions; these conditions will be sets and so will be partially ordered by $\subset$ and we insist that the minimal element, $\phi$, be a member of $C$. We also have a class $T$, the members of which we call "terms;" finally there is a one-one mapping $x \mapsto \mathrm{x}$ of the class of all sets into $T$. For every formula $\mathfrak{A}\left(x_{1}, \ldots, x_{n}\right)$ of our language, with no free variables other than $x_{1}, \ldots, x_{n}$, we suppose given 
a subclass $\Vdash_{\mathfrak{Y}}$ of $C \times T^{n}$. We write $p \Vdash \mathfrak{Y}\left(t_{1}, \ldots, t_{n}\right)$ for $\left\langle p, t_{1}, \ldots, t_{n}\right\rangle \in \Vdash_{\mathfrak{M}}$, and we read this as " $p$ forces $\mathfrak{A}\left(t_{1}, \ldots, t_{n}\right)$." (By "forcing," we here mean what is frequently referred to as "weak forcing," i.e., forcing of the double negation.)

Although we will be using forcing relations rather than countable models, we will use the terminology of models to make the process more intuitive. We can do this since, if our understanding is that we are extending from a model $\mathfrak{M}$ to a model $\mathfrak{N}$, we are able to define in our language a unary predicate $S(x)$, which reads " $x$ is standard," which is satisfied in $\mathfrak{R}$ precisely by the members of $\mathfrak{M}$. In $\mathfrak{R}$, each set is denoted by some $t \epsilon T$. In particular, if $x \in \mathfrak{M} \subset \mathfrak{N}, x$ is denoted by the term $\mathbf{x}$. If $\mathfrak{A}$ is a formula of the language of set theory, let $\mathfrak{g}^{S}$ be the formula obtained by relativizing the quantifiers of $S$; i.e.,

$$
\begin{aligned}
& {[\forall x \mathscr{B}(x)]^{\mathrm{S}} \equiv_{d f}\left[\forall x\left(\mathrm{~S}(x) \rightarrow \mathfrak{B}^{\mathrm{S}}(x)\right)\right] ;} \\
& {[\exists x \mathfrak{B}(x)]^{\mathrm{S}} \equiv_{d f}\left[\exists x\left(\mathrm{~S}(x) \wedge \mathfrak{B}^{\mathrm{S}}(x)\right)\right] ;}
\end{aligned}
$$

this relativization commutes with the logical connectives, and is the identity on atomic formulas. Then $\mathfrak{A}\left(x_{1}, \ldots, x_{n}\right)$ holds in the ground model $\mathfrak{M}$ if and only if $\mathfrak{I}^{\mathrm{S}}\left(\mathbf{x}_{1}, \ldots, \mathbf{x}_{n}\right)$ is true in the extension $\mathfrak{R}$.

In our use of forcing, we will be using sets of conditions (rather than classes) and we will obtain our extension model by adding a set a (or sets) of ordinals to our ground model $\mathfrak{M}$. For these reasons, by the results of [9] and [10], we need only specify the set $C$ of conditions and the action of our conditions on atomic formulas of the form $\alpha \in \boldsymbol{a}$ to insure that the classes $\Vdash_{\mathfrak{n}}$ will satisfy the following properties (taken directly from [11]):

(a) $p \Vdash \sim \mathfrak{Q}\left(t_{1}, \ldots, t_{n}\right)$ if and only if for no $q$ such that $p \subset q$ does $q \Vdash \mathfrak{A}\left(t_{1}, \ldots, t_{n}\right)$.

(b) $p \Vdash \mathfrak{A}\left(t_{1}, \ldots, t_{m}\right) \vee \mathfrak{B}\left(t_{1}, \ldots, t_{m}\right)$ if and only if for every $q$ such that $p \subset q$ there is a $q^{\prime}$ such that $q \subset q^{\prime}$ and either $q^{\prime} \Vdash \mathfrak{H}\left(t_{1}, \ldots, t_{m}\right)$ or $q^{\prime} \Vdash \mathfrak{B}\left(t_{1}, \ldots, t_{m}\right) ; p \Vdash \exists x \mathfrak{A}\left(t_{1}, \ldots, t_{n}, x\right)$ if and only if for every $q$ such that $p \subset q$ there is a $q^{\prime}$ such that $q \subset q^{\prime}$ and a $t \in T$ such that $q^{\prime} \Vdash \mathfrak{A}\left(t_{1}, \ldots, t_{n}, t\right)$.

(c) $p \Vdash \mathfrak{U}\left(t_{1}, \ldots, t_{n}\right) \wedge \mathfrak{B}\left(t_{1}, \ldots, t_{n}\right)$ if and only if $p \Vdash \mathfrak{U}\left(t_{1}, \ldots, t_{n}\right)$ and $p \Vdash \mathfrak{B}\left(t_{1}, \ldots, t_{n}\right) ; p \Vdash \mathfrak{A}\left(t_{1}, \ldots, t_{n}, x\right)$ if and only if for every $t \in T$, $p \Vdash \mathfrak{A}\left(t_{1}, \ldots, t_{n}, t\right)$.

(d) $p \Vdash S(t)$ if and only if for every $q$ such that $p \subset q$ there is a $q^{\prime}$ such that $q \subset q^{\prime}$ and a set $x$ such that $q^{\prime} \Vdash t=x . p \Vdash t \epsilon \mathrm{b}$ if and only if for every $q$ such that $p \subset q$ there is a $q^{\prime}$ such that $q \subset q^{\prime}$ and a set $x \in b$ such that $q^{\prime} \Vdash t=x$.

(e) Let $\mathfrak{A}\left(x_{1}, \ldots, x_{n}\right)$ and $\mathfrak{B}\left(x_{1}, \ldots, x_{k}\right)$ be formulas of our language. Let $t_{1}, \ldots, t_{n}, s_{1}, \ldots, s_{k}$ be terms from $T$ such that that sentences $\mathfrak{A}\left(t_{1}, \ldots, t_{n}\right)$ and $\mathfrak{B}\left(s_{1}, \ldots, s_{k}\right)$ coincide. Then $p \Vdash \mathfrak{H}\left(t_{1}, \ldots, t_{n}\right)$ if and only if $p \Vdash \mathfrak{B}\left(s_{1}, \ldots, s_{k}\right)$.

(f) If $\mathfrak{A}\left(x_{1}, \ldots, x_{n}\right)$ is an axiom of logic or ZF then $p \Vdash \mathfrak{A}\left(t_{1}, \ldots, t_{n}\right)$. If $p \Vdash \mathfrak{A}\left(t_{1}, \ldots, t_{n}\right)$ and $p \Vdash \mathfrak{A}\left(t_{1}, \ldots, t_{n}\right) \rightarrow \mathfrak{B}\left(t_{1}, \ldots, t_{n}\right)$ then $p \Vdash \mathfrak{B}\left(t_{1}, \ldots, t_{n}\right)$. Hence if $\mathfrak{A}\left(x_{1}, \ldots, x_{n}\right)$ is a theorem of ZF then $p \mathbb{R} \mathfrak{A}\left(t_{1}, \ldots, t_{n}\right)$.

Note that if $\mathfrak{A}\left(t_{1}, \ldots, t_{n}\right)$ and $\mathfrak{B}\left(t_{1}, \ldots, t_{n}\right)$ contradict one another in $\mathbf{Z F}$ 
then we cannot have $p \Vdash \mathfrak{A}\left(t_{1}, \ldots, t_{n}\right)$ together with $p \Vdash \mathfrak{B}\left(t_{1}, \ldots, t_{n}\right)$. Suppose we did have both. Then from $p \Vdash \mathfrak{A}\left(t_{1}, \ldots, t_{n}\right)$ and $p \Vdash \mathfrak{A}\left(t_{1}, \ldots, t_{n}\right)$ $\rightarrow \sim \mathfrak{B}\left(t_{1}, \ldots, t_{n}\right)$ (which we have by $(\mathrm{f})$ ) we get $p \Vdash \sim \mathfrak{B}\left(t_{1}, \ldots, t_{n}\right)$, and this contradicts $p \Vdash B B\left(t_{1}, \ldots, t_{n}\right)$, by (a).

(g) $\phi \Vdash \forall x(x$ is an ordinal $\rightarrow \mathrm{s}(x))$.

The following are provable from (a) - (f):

(h) If $p \Vdash \mathfrak{A}\left(t_{1}, \ldots, t_{n}\right)$ and $p \subset q$, then also $q \Vdash \mathfrak{A}\left(t_{1}, \ldots, t_{n}\right)$.

(i) $p \Vdash \mathfrak{A}\left(t_{1}, \ldots, t_{n}\right) \rightarrow \mathfrak{B}\left(t_{1}, \ldots, t_{n}\right)$ if and only if for every $q$ such that $p \subset q$ and such that $q \Vdash \mathfrak{A}\left(t_{1}, \ldots, t_{n}\right)$ there is a $q^{\prime}$ such that $q \subset q^{\prime}$ and $q^{\prime} \Vdash \mathfrak{B}\left(t_{1}, \ldots, t_{n}\right)$.

(j) $\quad p \Vdash \forall x\left(S(x) \rightarrow \mathfrak{A}\left(t_{1}, \ldots, t_{n}, x\right)\right)$ if and only if for every $x, p \Vdash \mathfrak{A}\left(t_{1}, \ldots\right.$, $\left.t_{n}, x\right) ; p \Vdash \forall x\left(x \in \mathrm{b} \rightarrow \mathfrak{A}\left(t_{1}, \ldots, t_{n}, x\right)\right)$ if and only if for every $x \in b, p \Vdash \mathfrak{H}\left(t_{1}, \ldots\right.$, $\left.t_{n}, \mathbf{x}\right)$. Use a similar definition for existential quantifiers.

We say that a formula $\mathscr{Q}\left(x_{1}, \ldots, x_{n}\right)$ of the language of ZF with no free variables other than $x_{1}, \ldots, x_{n}$ is absolute with respect to the extension if for all $x_{1}, \ldots, x_{n}$

$$
\phi \Vdash \mathfrak{A}\left(\mathbf{x}_{1}, \ldots, \mathbf{x}_{n}\right) \text { if } \mathfrak{A}\left(x_{1}, \ldots, x_{n}\right)
$$

and

$$
\phi \Vdash \sim \mathfrak{A}\left(\mathbf{x}_{1}, \ldots, \mathbf{x}_{n}\right) \text { if } \sim \mathscr{U}\left(x_{1}, \ldots, x_{n}\right) .
$$

It can be verified that the following formulas are absolute:

(k) $x \in y, x=y, x \subset y, x \cap y=\phi,\{x\}=y, x=y \cup z, \bigcup_{x}=y, f$ is a function from $x$ into $y, f$ is a function from $x$ onto $y, f$ is a one-one function from $x$ onto $y, f$ is a function from $x$ into $\boldsymbol{P}(y), f$ is a one-one function from $x$ into $\boldsymbol{p}(y), z=\bigcup f(x), x$ is an ordinal.

\section{CHAPTER I}

\section{CONSTRUCTIBLE SETS OF INTEGERS}

It is clear from [8] that every integer is constructible. It is equally easy to prove that any finite set of integers is constructible. The question then arises: "What happens if we assume that every set of integers is constructible?" (We abbreviate the proposition "every set of integers is constructible", by $\boldsymbol{P}(\omega) \subset \mathrm{L}$.). We can immediately conclude that the assumption that $\boldsymbol{p}(\omega) \subset \mathrm{L}$ does not contradict anything in $Z F$, since we have:

Theorem I.1 If ZF is consistent then $\mathbf{Z F}+\boldsymbol{P}(\omega) \subset \mathrm{L}$ is consistent.

Proof: This is clear, since we know that if $Z F$ is consistent then so is $Z F+V=L$. Since $\boldsymbol{P}(\omega) \subset L$ is a theorem in $Z F+V=L$, our theorem is proved. 
We next want to know whether the assumption $\boldsymbol{P}(\omega) \subset \mathrm{L}$ adds anything new to ZF set theory. We answer this in the affirmative since:

Theorem I.2 $\boldsymbol{P}(\omega) \subset \mathrm{L}$ is not provable in $\mathbf{Z F}+\mathbf{G C H}$.

This follows immediately from Cohen's proof of the independence of $V=L$. In his proof, he found a model of $Z F$ in which $G C H$ holds, but in which there is a non-constructible subset of $\omega$.

On the positive side, Cohen has shown, in [3], that

Theorem I.3 ZF $+\boldsymbol{P}(\omega) \subset \mathrm{L} \rightarrow \mathrm{CH}$.

Proof: We found the proof in [3] difficult to follow, so we will present a proof of this in detail. The proof of Theorem I.3 will follow closely the method that Doss [4] used to show that $V=L$ implies $G C H$. We will use his notation and numbering for this proof. His theorems will be stated without proof and in smaller type, if the proof of the theorem goes through unchanged under the weakened assumption.

11.8 Definition. $\langle y x\rangle \in$ As $. \equiv: y$ is constructible. $y \in x . \quad(z)\left[\operatorname{Od}^{\prime} z<\operatorname{Od}^{\prime} y . \supset\right.$. $\sim(z \in X)]$. Rel (As).

Note that we had to add the condition that $y$ be constructible to the right hand side of the equivalence.

11.81 Definition. $C^{\prime} \alpha=\operatorname{Od}^{\prime}\left[\mathrm{As}^{\prime}\left(\mathrm{F}^{\prime} \alpha\right)\right]$. C $\quad \Im_{\mathfrak{n}}$ On.

11.82 Definition. $C_{1}{ }^{\prime} \alpha=\operatorname{Od}^{\prime}\left[\operatorname{As}^{\prime}\left(F^{\prime} \alpha-F^{\prime} C^{\prime} \alpha\right)\right] . \quad C_{1} \Im_{n} O n$.

12.1 Definition. If $m \subset \mathrm{On}$ and $m$ is closed with respect to $C, C_{1}, \mathrm{~K}_{1}, \mathrm{~K}_{2}$ and with respect to $\mathrm{J}_{0}, \ldots, \mathrm{J}_{8}$ as triadic relations, define recursively a function $\mathrm{H}$ on $\mathrm{On}$ as follows:

$$
\begin{aligned}
& \eta \in \mathfrak{B}\left(J_{0}\right) \cdot \supset \cdot \mathrm{H}^{\prime} \eta=\mathrm{H}^{\prime \prime}(m \cap \eta) \\
& \eta=J_{i}\langle\beta \gamma\rangle . \supset \cdot \mathrm{H}^{\prime} \eta=\mathfrak{F}_{i}\left(\mathrm{H}^{\prime} \beta, \mathrm{H}^{\prime} \gamma\right) \text { for } i=1, \ldots, 8 .
\end{aligned}
$$

12.11 If $\eta \in m$, then every element $x$ of $H^{\prime} \eta$ is of the form $H^{\prime} \alpha$ with $\alpha \epsilon m \eta$.

12.12 If $m$ satisfies the conditions of definition 12.1, then $\alpha \in m . \supset . \mathrm{Od}^{\prime} \mathrm{F}^{\prime} \alpha \in m$.

12.2 1) $\mathrm{F}^{\prime} \alpha \epsilon \mathrm{F}^{\prime} \eta . \equiv . \mathrm{H}^{\prime} \alpha \epsilon \mathrm{H}^{\prime} \eta$ for $\eta \epsilon m, \alpha \epsilon m \cap \eta$.

2) $F^{\prime} \alpha=F^{\prime} \eta$. $\equiv . H^{\prime} \alpha=H^{\prime} \eta$ for $\eta \epsilon m, \alpha \epsilon m \cap \eta$.

12.3 If $G$ is an isomorphism from $m$ to an ordinal $\zeta$ with respect to $\mathrm{E}$, then $\mathrm{H}^{\prime} \eta=$ $F^{\prime} G^{\prime} \eta$ for $\eta \in m$.

The following two theorems are slightly different than their corresponding numbers in [4].

$12.4 \overline{\overline{\mathrm{F}}^{\prime \prime} \omega_{\alpha}} \leqq \aleph_{\alpha}$

Proof: We note that:

(1) Od is a function from $F^{\prime \prime} \omega_{\alpha}$ into $\omega_{\alpha}$,

and

(2) Od is one-one 
since, if we have $\operatorname{Od}^{\prime} x=\operatorname{Od}^{\prime} y$, we have that $x$ and $y$ are both first constructed by the same ordinal number, say $\beta$. Hence we have $x=\mathrm{F}^{\prime} \beta=y$. By (1) and (2), our theorem is proved.

$12.52^{\aleph_{0}} \leqq \overline{\overline{F^{\prime \prime} \omega_{1}}}$

Proof: Since $\overline{\overline{\boldsymbol{p}(\omega)}}=2^{\aleph_{0}}$, it is sufficient to show that

(1) $\boldsymbol{p}(\omega) \subset F^{\prime \prime} \omega_{1}$.

(2) Suppose that $u \in \boldsymbol{P}(\omega)$.

Since $\boldsymbol{P}(\omega) \subset L$, there is some ordinal number $\delta$ such that

(3) $u=F^{\prime} \delta$.

By (2), we know that every element of $u$ is an integer. But every integer is constructible by a finite ordinal. Hence, we have

(4) $u \subset F^{\prime \prime} \omega$.

Now form the closure of the set $\omega \cup\{\delta\}$ with respect to $C, C_{1}, K_{1}, K_{2}$ and with respect to the $J_{i}, i=0, \ldots, 8$, as triadic relations, according to 8.73 of Gödel [8], and let the closure be denoted by $m$. (Note, the closure $x^{*}$ of a set $x$ with respect to a triadic relation $R$ is the smallest class including $x$ which is closed with respect to $R$, i.e., $R^{\prime \prime} x \subset x^{*}$.) Now, by $8.73, m$ is a set and

(5) $\bar{m}=\aleph_{0}$.

This is true even without $A C$ since $\omega \cup\{\delta\}$ is a countable set. Since $m$ is a set of ordinal numbers, it is well-ordered by $E$ (concerning definition of $E$, see 7.161 of [8]) and hence it is isomorphic to some ordinal $\zeta$. Let the isomorphism be denoted by $G$, so that we have:

(6) $G^{\prime \prime} m=\zeta$.

Hence, we have

(7) $\overline{\bar{\zeta}}=\overline{\bar{m}}=\aleph_{0}$.

By 12.3 , since $\delta \epsilon m$, we have

(8) $H^{\prime} \delta=F^{\prime} G^{\prime} \delta$.

Hence, by (8) and (7), respectively,

(9) $\operatorname{Od}^{\prime}\left(H^{\prime} \delta\right) \leqq G^{\prime} \delta<\omega_{1}$.

We know that $\omega \subset m$, and by 12.1 ,

(10) $F^{\prime} \beta=H^{\prime} \beta$

for $\beta \epsilon \omega$. We may suppose that

(11) $\delta \geqq \omega$,

otherwise there is nothing to prove. Therefore, by 12.2 , for all $\beta \epsilon \omega$,

(12) $F^{\prime} \beta \epsilon F^{\prime} \delta . \equiv . H^{\prime} \beta \epsilon H^{\prime} \delta$. 
Hence, $F^{\prime} \delta$ and $H^{\prime} \delta$ have exactly the same elements in common with $F^{\prime \prime} \omega$; i.e.,

(13) $F^{\prime} \delta \cap F^{\prime \prime} \omega=H^{\prime} \delta \cap F^{\prime \prime} \omega$.

But, by (3) and (4), we have

(14) $F^{\prime} \delta \subset F^{\prime \prime} \omega$.

Therefore, we have

(15) $F^{\prime} \delta=H^{\prime} \delta \cap F^{\prime \prime} \omega$.

By 9.27 of [8], we have,

(16) $\omega$ is in the range of $J_{0}$.

Hence, by 9.35 of [8],

(17) $F^{\prime \prime} \omega=F^{\prime} \omega$

and hence, by (15)

(18) $F^{\prime} \delta=H^{\prime} \delta \cap F^{\prime} \omega$.

(We did not need $A C$ for 9.27 and 9.35 of [8], since we are dealing with $\omega$. ) Therefore, by 9.611 of [8], (18), and (9), we have

(19) $\operatorname{Od}^{\prime} u<\omega_{1}$.

But this is the same as saying

(20) $u \in \mathrm{F}^{\prime \prime} \omega_{1}$.

Hence we have $\boldsymbol{P}(\omega) \subset F^{\prime \prime} \omega_{1}$, which is what we wanted to prove.

The proof of Theorem I.3 now follows quite simply. By 12.4 and 12.5, we have $2^{\aleph_{0}} \leqq \aleph_{1}$. But Od well-orders $P(\omega)$, hence $2^{\aleph_{0}}=\aleph_{\alpha}$ for some $\alpha \leqq 1$. Since $\aleph_{0}<2^{\aleph_{0}}$, we have $2^{\aleph_{0}}=\aleph_{1}$.

Note: This proof was carried out in Gödel-Bernays set theory in order to take advantage of Doss's results. It could also have been done in ZF if it had been so desired, since every theorem in Gödel-Bernays set theory that deals only with sets is also a theorem of ZF. (See Cohen [3], pp. 77-78.)

We list one more result which was proved first by Silver [21] and then elaborated upon by Solovay [22].

Theorem I.4 The existence of a Ramsey cardinal is incompatible with $P(\omega) \subset L$.

Since every measurable cardinal is also a Ramsey cardinal, we have:

Corollary. The existence of a measurable cardinal is incompatible with $\boldsymbol{p}(\omega) \subset L$.

This had earlier been proved directly by Gaifman [6] and Rowbottom [14].

In each of these past theorems, we note that they would remain true if 
we had used " $V=L$ " instead of "P $(\omega) \subset L$," so it seems that, in some respects, $\boldsymbol{P}(\omega) \subset L$ is "nearly as strong" as $V=L$. We now give some new results that show that this is not so.

Theorem I.5 $\boldsymbol{p}(\omega) \subset \mathrm{L}$ does not imply $\vee=\mathrm{L}$.

Proof: J. H. Silver in [21], mentions a model of ZF + AC given by Karel Prikry in which every countable set of ordinal numbers is constructible but in which there is a non-constructible subset of $\omega_{1}$. Since every set of integers is countable, this model suffices for the proof of the theorem. Here, however, since the model of Prikry is not yet published and, therefore, is unknown to us, we will prove the theorem directly.

It suffices to find a model in which $\boldsymbol{P}(\omega) \subset L$ but $\vee \neq L$. We will start with the minimal model $\mathfrak{M}$, (in which $V=L$ holds). (See Cohen [3].) We must then define $C$, the set of conditions.

Definition I.5.1 Let $C$ be the set of all at most countable sets of statements of the form " $\alpha \epsilon a$ " or " $~(\alpha \epsilon a)$ ", where $\alpha$ is an ordinal $<\omega_{1}$, a is a formal symbol of the language and not both " $\alpha \in a$ ", and " $~(\alpha \in a)$ ", occur in the same set.

We now must determine the action of the forcing conditions on our atomic formulas.

Definition I.5.2 For $p \in \mathrm{C}$, we define $p \Vdash \alpha \in \mathrm{a}$ if and only if " $\alpha \epsilon \mathrm{a}$ " is in $p$.

Since $\mathfrak{M}$ is countable, there are at most countably many formulas in $\mathfrak{M}$. Hence, we can find an infinite sequence (called a complete sequence) of conditions, $\left\{p_{n}\right\}_{n<\omega}$, such that for every formula $\mathfrak{A}$, there is some $k$ such that either $p_{k} \Vdash \mathfrak{A}$ or $p_{k} \Vdash \sim \mathfrak{A}$.

Definition I.5.3 Let $\left\{p_{n}\right\}_{n<\omega}$ be a complete sequence of forcing conditions; we define: $\mathbf{a}=\left\{\alpha \mid \exists k\right.$ such that $\left.p_{k} \Vdash \alpha \epsilon a\right\}$.

Our extension $\mathfrak{R}$ is now a model of $\mathbf{Z F}$ that contains $\mathfrak{M}$ and which has $a$ as an element. We also note that the true statements in $\mathfrak{A}$ are precisely those which are forced by some $p_{k}$. We now state without proof a very important lemma which was first proved by Solovay and which occurs as Lemma 7 of [11]. Here, this lemma is changed slightly from its original presentation in [11]. There, the authors assume $A C$ at all times, and hence, all infinite cardinals are initial ordinals.

Lemma I.5.4 Let $\lambda$ be an initial ordinal. Suppose that the set of conditions has the property that every well-ordered increasing sequence of conditions of length at most $\lambda$ has an upper bound in $C$. Then $\forall x(x \cap \lambda$ is standard $)$ holds in the Cohen extension.

Lemma I.5.5 In $\mathfrak{R}, \boldsymbol{p}(\omega) \subset \mathrm{L}$.

Proof: Suppose we have an infinite sequence of conditions $\left\{p_{n}\right\}_{n<\omega}$ such that

(1) $p_{0} \subset p_{1} \subset p_{2} \ldots$

Now let 
(2) $p=\bigcup_{n<\omega} p_{n}$.

Since each $p_{n}$ is at most countable, we know from (2) that $p$ is countable. Likewise $p$ does not contain both " $\alpha \in a$ " and " $~(\alpha \in a)$ " for any $\alpha$ since otherwise, by (1), these statements would both have to be in some $p_{n}$, in which case $p_{n}$ would not be a condition. Therefore

(3) $p$ is a condition.

It is also clear by (2) that

(4) $p$ is an upper bound for the sequence $\left\{p_{n}\right\}_{n<\omega}$.

Now, by (3) and (4), C satisfies the conditions of Lemma I.5.4 where $\lambda=\omega$. Therefore, in $\mathfrak{R}$, we have

(5) $\forall x(x \cap \omega$ is standard $)$.

Therefore, in $\mathfrak{R}$,

(6) every subset of $\omega$ is standard.

Hence, by definition of "standard,"

(7) every subset of $\omega$ in $\mathfrak{N}$ is also in $\mathfrak{M}$.

But $V=L$ holds in $\mathfrak{M}$, hence

(8) every subset of $\omega$ is constructible in $\mathfrak{M}$.

But by point $(\mathrm{k})$ of our Introduction, the ordinal numbers of $\mathfrak{M}$ and $\mathfrak{R}$ are the same. But since the constructible sets depend only on the ordinal numbers, we have

(9) the constructible sets in $\mathfrak{M}$ are constructible in $\mathfrak{N}$.

Hence, we have,

(10) every subset of $\omega$ is constructible in $\mathfrak{R}$.

This is exactly what we wanted to prove.

We now wish to show that $V=L$ does not hold in our extension model $\Re$.

Lemma I.5.6 a is not constructible in $\Re$.

Proof: As in point (9) of the previous lemma, $\mathfrak{M}$ and $\mathfrak{R}$ have the same constructible sets. Therefore, if $a$ is not standard, it is not in $\mathfrak{M}$, hence not constructible in $\mathfrak{R}$. Therefore we need only show:

(1) a is not standard.

To show (1) we need only show that if $t \in \mathfrak{M}$, then

(2) $p \in C \rightarrow p \Vdash \sim(\mathbf{a}=\mathbf{t})$.

Hence, by (a) of the Introduction, we must show that for no $q$ such that $p \subset q$ do we have $q \Vdash a=t$. Now suppose we have

(3) $p \in C$ 
(4) $p \subset q$.

Let $\alpha$ be the smallest ordinal number such that neither " $\alpha \epsilon a$ " nor " $\sim(\alpha \epsilon a)$ "' is in $q$. Such an $\alpha$ exists since $q$ is at most countable and there are $\aleph_{1}$ ordinals less than $\omega_{1}$.

(5) If $\alpha \in t$, let $q^{\prime}=q \cup\{$ " $\sim(\alpha \in \mathrm{a})$ ', $\}$.

(6) If $\sim(\alpha \in t)$, let $q^{\prime \prime}=q \cup\{$ " $\alpha \in \mathrm{a}$ "' $\}$.

Therefore, if $\alpha \epsilon t$, by (5), we have

(7) $q^{\prime} \Vdash \alpha \in \mathbf{t} \wedge \sim(\alpha \in \mathbf{a})$.

If we do not have $\alpha \epsilon t$, then, by (6),

(8) $q^{\prime \prime} \Vdash \sim(\alpha \in \mathbf{t}) \wedge \boldsymbol{\alpha} \in \mathbf{a}$.

Since either (7) and (8) must apply in any situation, $q$ does not force $\mathbf{a}=\mathbf{t}$. Therefore, we have verified (2), and the lemma is proved. This completes the proof of Theorem I.5. We now strengthen Theorem I.5 to the following:

Theorem I.6 $\boldsymbol{p}(\omega) \subset \mathrm{L}$ does not imply $\mathbf{G C H}$.

Proof: As in the proof of Theorem I.5, we will find a model of ZF in which $\boldsymbol{P}(\omega) \subset \mathrm{L}$ holds but in which $\mathrm{GCH}$ fails. Again we start with the minimal model $\mathfrak{M}$ and we construct an extension model $\mathfrak{R}$. As before, we must define C, our set of conditions:

Definition I.6.1 Let $C$ be the set of all at most countable statements of the form " $\alpha \epsilon \mathrm{a}_{\delta}$ ", or " $\sim\left(\alpha \epsilon \mathrm{a}_{\delta}\right)$ " for $\alpha<\omega_{1}, \delta<\omega_{\tau}$, where $\tau$ is an ordinal number $>2$, and such that for no $\alpha, \delta$ do we have both " $\alpha \in \mathrm{a}_{\delta}$ " and " $\sim\left(\alpha \in \mathrm{a}_{\delta}\right)$ " in the same set.

We again specify the action of the forcing conditions on our atomic formulas:

Definition I.6.2 For $p \in \mathrm{C}$, we define $p \Vdash \alpha \epsilon \mathrm{a}_{\delta}$ if " $\alpha \epsilon \mathrm{a}_{\delta}$ " $\epsilon p$.

Definition I.6.3 Let $\left\{p_{n}\right\}_{n<\omega}$ be a complete sequence of forcing conditions. For each $\delta<\omega_{\tau}$, let $\mathbf{a}_{\delta}=\left\{\alpha \mid \exists k\right.$ such that $\left.p_{k} \Vdash \alpha \epsilon \mathrm{a}_{\delta}\right\}$.

Lemma I.6.4 $\boldsymbol{p}(\omega) \subset \mathrm{L}$ is true in $\mathfrak{R}$.

Proof: We need only show that $C$ satisfies the hypotheses of Lemma I.5.4 where $\lambda=\omega$. This is true by exactly the same proof as Lemma I.5.5.

Lemma I.6.5 $\sim\left(\delta=\delta^{\prime}\right) \rightarrow \sim\left(\mathbf{a}_{\delta}=\mathbf{a}_{\delta^{\prime}}\right)$.

Proof: Suppose

(1) $\sim\left(\delta=\delta^{\prime}\right)$.

We need only show that

(2) $p \in C \rightarrow p \Vdash \sim\left(\mathbf{a}_{\delta}=\mathbf{a}_{\delta^{\prime}}\right)$.

By (a) of the Introduction, we must show that for no $q$ such that $p \subset q$ do we have $q \Vdash \mathbf{a}_{\delta}=\mathbf{a}_{\delta^{\prime}}$. Now suppose we have 
(3) $p \in \mathrm{C}$

(4) $p \subset q$.

Since $q$ is a condition, it is at most countable, hence there is a condition $q^{\prime}$ such that $q \subset q^{\prime}$ and there is some $\alpha \epsilon \omega_{1}$ such that " $\alpha \epsilon \mathrm{a}_{\delta}$ " and " $\sim\left(\alpha \in \mathrm{a}_{\delta^{\prime}}\right)$ ", are both in $q^{\prime}$. Therefore, we have

(5) $q^{\prime} \Vdash \alpha \in \mathbf{a}_{\delta}$

(6) $q^{\prime} \Vdash \sim\left(\alpha \in \mathbf{a}_{\delta^{\prime}}\right)$.

Therefore, by (5), (6) and (c) of the Introduction,

(7) $q^{\prime} \Vdash \alpha \in \mathbf{a}_{\delta} \wedge \sim\left(\alpha \in \mathbf{a}_{\delta^{\prime}}\right)$.

Therefore, $q$ does not force $\mathbf{a}_{\delta}=\mathbf{a}_{\delta^{\prime}}$, hence $p \Vdash \sim\left(\mathbf{a}_{\delta}=\mathbf{a}_{\delta^{\prime}}\right)$.

We can now prove Theorem I.6. We know that for every $\delta<\omega_{\tau}$ we have $\mathbf{a}_{\delta} \subset \omega_{1}$. Since, by $(\mathrm{k})$ of the Introduction, cardinalities are preserved in the extension, we have $\aleph_{\tau} \leqq 2^{\aleph_{1}}$. But this contradicts $G C H$, and hence we have Theorem I.6.

We now strengthen Theorem I.5 and Theorem I.6 even further:

Theorem I.7 $\boldsymbol{p}(\omega) \subset \mathrm{L}$ does not imply AC.

Proof: The proof of this theorem will parallel that of Cohen [3]. We will proceed as in the proof of our two previous theorems.

Definition I.7.1 Let $C$ be the set of all at most countable sets of statements of the form " $\alpha \epsilon \mathrm{a}_{\delta}$ " or " $\sim\left(\alpha \epsilon \mathrm{a}_{\delta}\right)$ " for $\alpha, \delta<\omega_{1}$, such that not both " $\alpha \in \mathrm{a}_{\delta}$ ", and " $\sim\left(\alpha \in \mathrm{a}_{\delta}\right)$ " occur in the same set for any $\alpha$ or $\delta$.

We now introduce a new constant, $W$, and so must specify the action of our forcing conditions on $W$.

Definition 1.7 .2

(i) For $p \epsilon \mathrm{C}, p \Vdash \alpha \epsilon \mathrm{a}_{\delta} \leftrightarrow$ " $\alpha \epsilon \mathrm{a}_{\delta}$ " $\epsilon p$

(ii) $p \Vdash a_{\delta} \in W \leftrightarrow p \in C$ and $\delta<\omega_{1}$.

Definition I.7.3 Let $\left\{p_{n}\right\}_{n<\omega}$ be a complete sequence of forcing conditions. Then:

(i) For $\delta<\omega_{1}, \mathbf{a}_{\delta}=\left\{\alpha \mid \exists k\right.$ such that $\left.p_{k} \Vdash \alpha \epsilon \mathbf{a}_{\delta}\right\}$ :

(ii) $\mathbf{W}=\left\{\boldsymbol{a}_{\delta} \mid \exists k\right.$ such that $\left.p_{k} \Vdash a_{\delta} \in \mathrm{W}\right\}$.

Hence, by Definition I.7.2, we have $\mathbf{W}=\left\{\mathbf{a}_{\delta} \mid \delta<\omega_{1}\right\}$.

Lemma I.7.4 $\boldsymbol{P}(\omega) \subset \mathrm{L}$ is true in $\mathfrak{R}$.

Proof: This follows by exactly the same method as Lemma I.5.5.

Definition I.7.5 Let $g$ be the group of all permutations $\pi$ of $\omega_{1}$ such that $\sim(\pi(\alpha)=\alpha)$ for only countably many $\alpha$; let $g_{\alpha}$ be the subgroup of $g$ defined by: $g_{\alpha}=\{\pi \mid \pi(\beta)=\beta$ for all $\beta \leqq \alpha\}$.

If $\pi$ is a permutation in $g$, we extend to a permutation of $\mathfrak{N}$ in the following way: 


\section{Definition I.7.6}

(i) $\pi(\mathbf{t})=\mathbf{t}$ for $\pi \in \mathrm{g}, t \in \mathfrak{M}$.

(ii) $\pi\left(\mathbf{a}_{\delta}\right)=\mathbf{a}_{\pi(\delta)}$ for $\pi \epsilon$ g, $\delta<\omega_{1}$.

(iii) $\pi(\mathbf{W})=\mathbf{W}$ for $\pi \in g$.

(iv) For $t \in \mathfrak{M}, \pi \in \mathfrak{g}$, if $t$ corresponds to the formula $\mathfrak{A} \leftrightarrow \mathscr{B}\left(t_{1}, \ldots, t_{n}\right)$, where $t_{i} \in \mathfrak{R}$, then $\pi(\mathfrak{A}) \longleftrightarrow \mathscr{B}\left(\pi\left(t_{1}\right), \ldots, \pi\left(t_{n}\right)\right)$.

(v) If $p$ is a forcing condition, $\pi(p)$ is the forcing condition defined by " $\alpha \epsilon \mathrm{a}_{\delta}$ " $\epsilon p \leftrightarrow$ " $\alpha \in \mathrm{a}_{\pi(\delta)}$ " $\epsilon \pi(p)$ and " $\sim\left(\alpha \epsilon \mathrm{a}_{\delta}\right)$ " $\epsilon p \leftrightarrow$ " $\sim\left(\alpha \epsilon \mathrm{a}_{\pi(\delta)}\right)$ "' $\epsilon \pi(p)$.

Lemma I.7.7 $p \subset q \leftrightarrow \pi(p) \subset \pi(q)$.

Proof: This follows easily from Definition I.7.6, (v).

Lemma I.7.8 $p \Vdash \mathfrak{A} \leftrightarrow \pi(p) \Vdash \pi(\mathfrak{A})$.

Proof: We use induction on formulas:

(1) Suppose $\mathfrak{A}=\sim \mathfrak{B}$. Then:

$$
\begin{aligned}
p \Vdash \mathfrak{A} & \leftrightarrow p \Vdash \sim \mathfrak{B} \\
& \leftrightarrow p \subset q \rightarrow q \text { does not force } \mathfrak{B} \\
& \leftrightarrow p \subset q \rightarrow \pi(q) \text { does not force } \pi(\mathfrak{B}) \\
& \leftrightarrow \pi(p) \subset \pi(q) \rightarrow \pi(q) \text { does not force } \pi(\mathfrak{B}) \\
& \leftrightarrow \pi(p) \Vdash \sim \pi(\mathfrak{B}) \\
& \leftrightarrow \pi(p) \Vdash \pi(\sim \mathfrak{B}) \\
& \leftrightarrow \pi(p) \Vdash \pi(\mathfrak{A})
\end{aligned}
$$

[by (a) of the Introduction] [by induction hypothesis] [by Lemma I.7.7] [by (a) of the Introduction] [by Definition I.7.6] [by (1)]

(2) Suppose $\mathfrak{A}=\mathfrak{B} \vee \mathfrak{D}$. Then:

$$
\begin{aligned}
p \Vdash \mathfrak{A} & \leftrightarrow p \Vdash \mathfrak{B} \vee \mathfrak{D} . \\
& \leftrightarrow p \Vdash \mathfrak{B} \text { or } p \Vdash \mathfrak{D} \\
& \leftrightarrow \pi(p) \Vdash \pi(\mathfrak{B}) \text { or } \pi(p) \Vdash \pi(\mathfrak{D}) \\
& \leftrightarrow \pi(p) \Vdash \pi(\mathfrak{B}) \vee \pi(\mathfrak{D}) \\
& \leftrightarrow \pi(p) \Vdash \pi(\mathfrak{B} \vee \mathfrak{D}) \\
& \leftrightarrow \pi(p) \Vdash \pi(\mathfrak{A})
\end{aligned}
$$

[by (b) of the Introduction] [by induction hypothesis] [by (b) of the Introduction] [by Definition I.7.6] [by (2)]

(3) Suppose $\mathfrak{A}=\exists x \mathfrak{B}(x)$. Then:

$p \Vdash \mathfrak{A} \leftrightarrow p \Vdash \exists x \mathfrak{B}(x)$

$\leftrightarrow p \subset q \rightarrow \exists q^{\prime}$ such that $q \subset q^{\prime}$ and $\exists t$ such that $q^{\prime} \Vdash \mathfrak{B}(t)$

[by (3) ]

[by (b) of the Introduction] $\leftrightarrow p \subset q \rightarrow \exists q^{\prime}$ such that $q \subset q^{\prime}$ and $\exists t$ such that $\pi\left(q^{\prime}\right) \Vdash \pi(B(t))$

[by induction hypothesis] $\leftrightarrow p \subset q \rightarrow \exists q^{\prime}$ such that $\pi(q) \subset \pi\left(q^{\prime}\right)$ and $\exists t$ such that $\pi\left(q^{\prime}\right) \Vdash \pi(\mathscr{B}(t))$

[by Lemma I.7.7] $\leftrightarrow p \subset q \rightarrow \exists \pi\left(q^{\prime}\right)$ such that $\pi(q) \subset \pi\left(q^{\prime}\right)$ and $\exists t$ such that $\pi\left(q^{\prime}\right) \Vdash \pi(\mathbb{B}(t))$ [since $\pi$ is a bijection] $\leftrightarrow \pi(p) \subset \pi(q) \rightarrow \exists \pi\left(q^{\prime}\right)$ such that $\pi(q) \subset \pi\left(q^{\prime}\right)$ and $\exists t$ such that $\pi\left(q^{\prime}\right) \Vdash \pi(\mathbb{B}(t))$

$\leftrightarrow \pi(p) \subset \pi(q) \rightarrow \exists \pi\left(q^{\prime}\right)$ such that $\pi(q) \subset \pi\left(q^{\prime}\right)$ and $\exists t$ such that

[by Lemma 1.7.7] $\leftrightarrow \pi(p) \Vdash \exists x \mathscr{B}(\pi(x))$

[by Definition I.7.6, (iv)] [by (b) of the Introduction] 


$$
\begin{aligned}
& \leftrightarrow \pi(p) \Vdash \exists \pi(x) \mathfrak{B}(\pi(x)\rangle \\
& \leftrightarrow \pi(p) \Vdash \pi(\mathfrak{Q})
\end{aligned}
$$

[since $\pi$ is a bijection]

(4) Suppose $\mathfrak{A}=\alpha \in \mathbf{a}_{\delta}$, or $\mathfrak{A}=\mathbf{a}_{\delta} \in \mathbf{W}$, or $\mathfrak{A}=\mathbf{t}_{1} \in \mathbf{t}_{2}, t_{1}, t_{2} \in \mathfrak{M}$. Then:

$p \Vdash \mathfrak{A} \leftrightarrow \pi(p) \Vdash \pi(\mathfrak{A})$

[by Definition I.7.6]

Lemma I.7.9 For each $t \in \mathfrak{R}$, formula $\mathfrak{A}$, and $p \in \mathcal{C}$, there is an $\alpha$ such that $\pi \in \mathfrak{g}_{\alpha} \rightarrow \pi(t)=t, \pi(\mathfrak{A})=\mathfrak{A}, \pi(p)=p$.

Proof: We will treat the three conditions separately:

(1) $t \in \mathfrak{R}$

If $t$ is standard, then $\pi(t)=t$.

If $t=\mathbf{a}_{\delta}$, then $\pi \epsilon g_{\delta}$ works, where $g_{\delta}$ is defined as in I.7.5.

If $t=\mathbf{W}$, then any $\pi$ works.

If $t$ corresponds to a formula $\mathfrak{A}\left(x, t_{1}, \ldots, t_{n}\right)$, then if there exist $\alpha_{i}$ such that $\pi \epsilon g_{\alpha_{i}}$ implies $\pi\left(t_{i}\right)=t_{i}$, then $\alpha=\max \left(\alpha_{i}\right)$ works, hence this case can be proved by induction on formulas.

(2) If $\mathfrak{A}$ is a statement, it can be handled in the same way as the last case of (1).

(3) If $p$ is a condition, then there is some $\alpha<\omega_{1}$ such that $\alpha$ is greater than any subscript of the $\alpha_{\delta}$ 's appearing in $p$.

If we now let $\alpha$ be the largest of those obtained in (1), (2), and (3), it will have the desired properties.

Lemma I.7.10 In $\mathfrak{R}, \mathbf{W}$ is a subset of $\boldsymbol{P}\left(\omega_{1}\right)$ such that $\mathbf{W}$ is uncountable and yet contains no subset of cardinality $\aleph_{1}$.

Proof: As in Lemma I.6.5, we have

(1) $\sim(\gamma=\delta) \rightarrow p \Vdash \sim\left(\boldsymbol{a}_{\gamma}=\boldsymbol{a}_{\delta}\right)$.

Since, in $\mathfrak{R}$, each $\boldsymbol{a}_{\delta} \in \mathbf{W}$, we have

(2) $\mathbf{W}$ is uncountable in $\mathfrak{R}$.

Now let $t \in \mathfrak{R}$ and assume that for some $p$ in $\left\{p_{n}\right\}_{n<\omega}$

(3) $p \Vdash\left\{t\right.$ is a $1-1$ function from $\omega_{1}$ into $\left.W\right\}$

Let $\zeta$ be an ordinal number such that $\zeta<\omega_{1}$ and if $g_{\zeta}$ is defined as in Definition I.7.5, then:

(4) $\pi \in g_{\zeta} \rightarrow \pi(t)=t$

and such that $\zeta$ is greater than any $\delta$ such that $a_{\delta}$ occurs in a statement of $p$. In the complete sequence $\left\{p_{n}\right\}_{n<\omega}$ there must be some $p^{\prime}$ such that $p \subset p^{\prime}$ and

(5) $p^{\prime} 1-t(\eta)=\mathbf{a}_{\sigma}$

for some $\eta$ and $\sigma$ where $\sigma$ is greater than $\zeta$, since $t$ takes uncountably many values. Let $\tau>\zeta$ be a countable ordinal such that $a_{\tau}$ does not appear in $p^{\prime}$ 
and let $\pi$ be the permutation which interchanges $\sigma$ and $\tau$ and is the identity on all other countable ordinals. If $p^{\prime \prime}=\pi\left(p^{\prime}\right)$, then, by (5),

(6) $p^{\prime \prime} \Vdash t(\eta)=a_{\tau}$.

We also have that, if $q=p^{\prime} \cup p^{\prime \prime}$, then

(7) $q$ is a forcing condition

since $p^{\prime}$ and $p^{\prime \prime}$ are identical except for conditions involving $a_{\sigma}$ and $a_{\tau}$ and $p^{\prime}$ does not involve $\sigma$ and $p^{\prime \prime}$ does not involve $\tau$. But $p \subset q$, therefore, by (3), $q$ forces $t$ to be a function. But, by (5),

(8) $q \Vdash t(\eta)=\mathbf{a}_{\sigma}$

and, by (6),

(9) $q \Vdash t(\eta)=\mathbf{a}_{\tau}$

and (8) and (9) give a contradiction.

Our theorem now follows quite easily. In $\mathfrak{N}, \mathbf{W}$ is a set such that $\overline{\bar{W}}>\aleph_{0}$ but $\sim\left(\aleph_{1} \leqq \overline{\bar{W}}\right)$, which violates $A C$. Thus, our theorem is proved.

This last theorem has some interesting consequences. In [1], Addison showed that the assumption of $V=L$ gives some strong results about projective well-orderings of the real numbers. In [13], we showed that the only lemma that Addison used that required $V=L$ for its proof is equivalent to $\boldsymbol{P}(\omega) \subset \mathrm{L}$. Therefore, by Theorem I.7, if we have $\boldsymbol{P}(\omega) \subset L$ we can get Addison's results in a system that does not even have $A C$.

We will now show how the results of this chapter fit in with previous findings. The references listed are not necessarily the original proofs, but were chosen for convenience. The phrases " $\mathscr{A} \rightarrow \mathfrak{B}$," “ $\mathscr{A} \not \mathfrak{B}$,", and " $\mathfrak{A}--\rightarrow \mathfrak{B}$," in the field of a given set theoretical system in which $\mathfrak{A}$ or $\mathfrak{B}$ are axioms, mean that, respectively, " $B$ is a consequence of $\mathfrak{A}, "$ " $B$ is not a consequence of $\mathfrak{A}$," and “ $\mathfrak{B}$ is not a consequence of $\mathfrak{A}$ nor is $\mathfrak{B}$ a consequence of $\sim \mathfrak{A} . "$ In the accompanying diagrams, if two propositions are not connected by any arrow, then either the connection is unknown or else the connection is obvious because of some intermediate proposition. We will first list some "classical" results, i.e., these were known before Cohen introduced his forcing technique.

(1) $V=L \rightarrow G C H$

(2) $\mathrm{GCH} \rightarrow \mathrm{AC}$

(3) $\mathrm{GCH} \rightarrow \mathrm{CH}$

[obvious ]

(4) $V=L \rightarrow(\sim(\exists$ measurable cardinal $))$

(5) $V=L \rightarrow \boldsymbol{p}(\omega) \subset \mathrm{L}$

[obvious]

(6) $\quad \mathrm{V}=\mathrm{L} \rightarrow$ Addison's results about projective sets

(7) $(\sim(\exists$ Ramsey cardinal $)) \rightarrow(\sim(\exists$ measurable cardinal $))$

[obvious]

(8) $\boldsymbol{p}(\omega) \subset \mathrm{L} \rightarrow \mathrm{CH}$ 


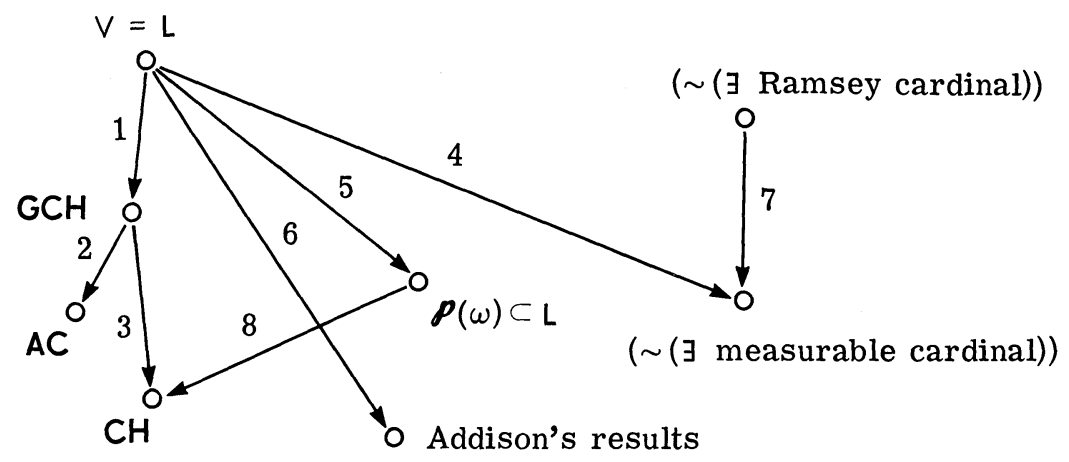

Next we will list some of Cohen's results together with some related results obtained since then.

(9) $\mathrm{GCH} \nrightarrow \mathrm{V}=\mathrm{L}$

(10) $\mathrm{GCH} \nrightarrow \boldsymbol{p}(\omega) \subset \mathrm{L}$

(11) $\mathrm{AC} \nrightarrow \mathrm{CH}$

(12) $\boldsymbol{p}(\omega) \subset L \rightarrow(\sim(\exists$ Ramsey cardinal $)$

(13) $\mathrm{GCH}-\rightarrow(\sim(\exists$ measurable cardinal $))$

(14) $\boldsymbol{P}(\omega) \subset \mathrm{L} \rightarrow$ Addison's Results

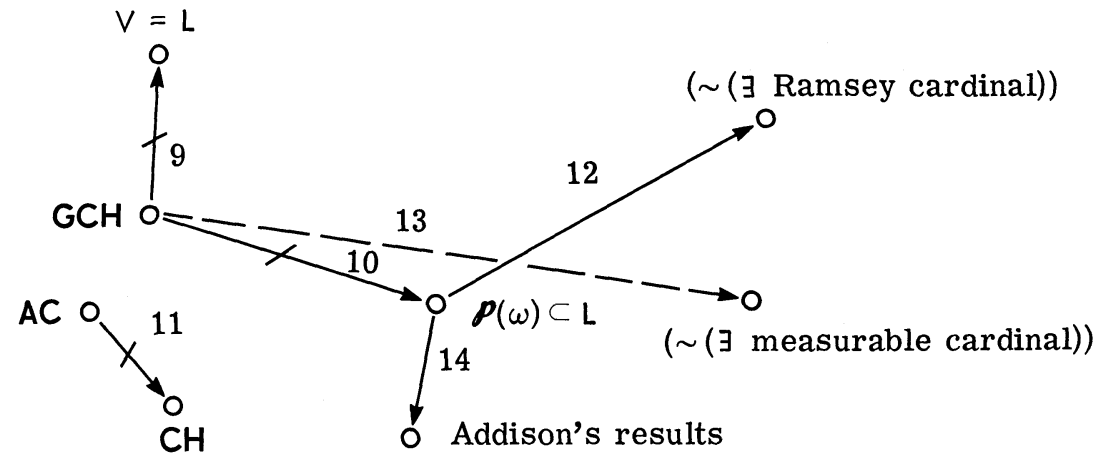

We now list the relevant results of Chapter I.

(15) $\boldsymbol{P}(\omega) \subset L \not f \vee=L$

(16) $P(\omega) \subset L f G C H$

(17) $\boldsymbol{p}(\omega) \subset L \neq A C$

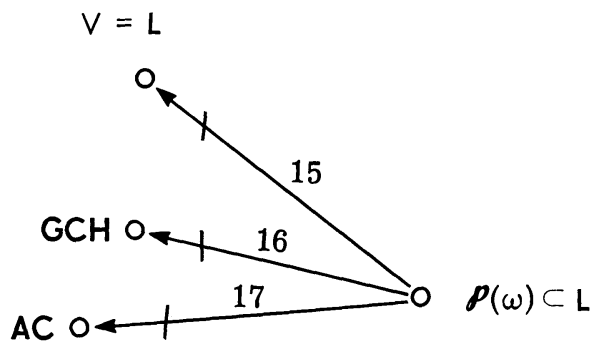


Combining the three diagrams and deleting any redundancies, we obtain the following:

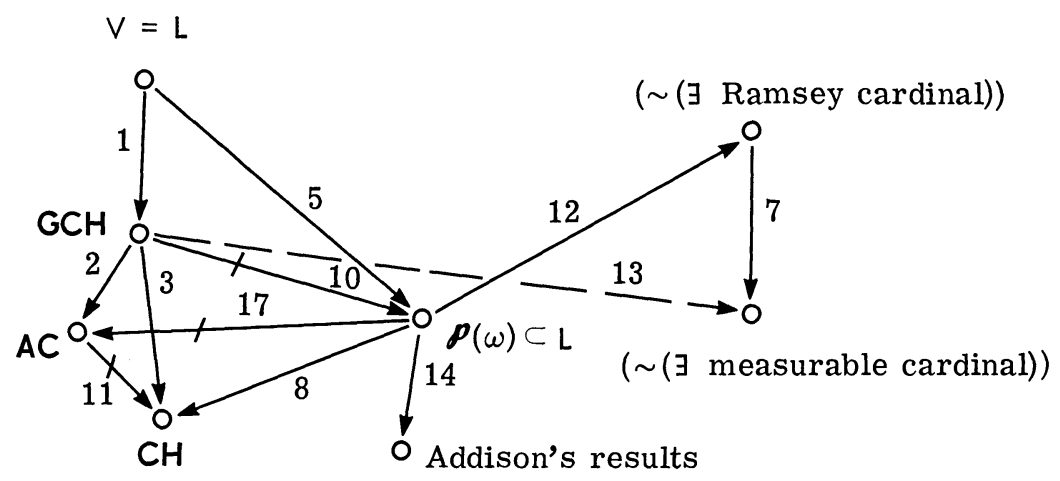

\section{CHAPTER II}

\section{CONSTRUCTIBLE SUBSETS OF ORDINALS}

We now generalize Chapter I and consider the propositions $\boldsymbol{P}(\alpha) \subset \mathrm{L}$, where $\alpha$ is an ordinal number. From the results of Chapter I, in particular Theorem I.1 and Theorem I.2, it can easily be seen that, for any ordinal number $\alpha, \boldsymbol{P}(\alpha) \subset \mathrm{L}$ is both consistent with ZF (if ZF itself is consistent) and, if $\alpha \geqq \omega$, it cannot be proved in ZF even with the use of $G C H$. We now consider the relationships between these propositions $\boldsymbol{P}(\alpha) \subset \mathrm{L}$ for various ordinal numbers $\alpha$. First, we note the fact that, if $\alpha<\beta$, then $\boldsymbol{P}(\alpha) \subset \boldsymbol{P}(\beta)$. This gives us the following easy theorem.

Theorem $\Pi$.1 If $\alpha<\beta$, then $\boldsymbol{P}(\beta) \subset \mathrm{L}$ implies $\boldsymbol{P}(\alpha) \subset \mathrm{L}$.

We next consider the situations in which we can infer $\boldsymbol{P}(\beta) \subset \mathrm{L}$ from $\boldsymbol{P}(\alpha) \subset \mathrm{L}$, where $\alpha<\beta$. A very simple case in which this can be done is where $\beta=\alpha+n$ where $n$ is a natural number. In fact, we have:

Theorem II.2 If $n<\omega$, then $\boldsymbol{P}(\alpha) \subset \mathrm{L}$ implies $\boldsymbol{P}(\alpha+n) \subset \mathrm{L}$.

Proof:

(1) Suppose we have $\boldsymbol{p}(\alpha) \subset$ L.

We will use induction on $n$. We first consider the case

(2) $n=0$.

In this case we have nothing to prove, since $\alpha+0=\alpha$.

(3) Suppose $P(\alpha+n) \subset \mathrm{L}$.

We now wish to show that $\boldsymbol{P}(\alpha+n+1) \subset \mathrm{L}$.

(4) Suppose $x \in \mathcal{P}(\alpha+n+1)$. 
Hence, we have

(5) $x \subset(\alpha+(n+1))$

but then

(6) $x \subset((\alpha+n)+1)$.

But this means, since for any ordinal $\gamma, \gamma+1=\gamma \cup\{\gamma\}$, that for every $z$,

(7) $z \epsilon x \rightarrow z \epsilon \alpha+n \cdot v \cdot z=\alpha+n$.

Hence, we have

(8) $x=y \cup w$

where $w=\phi$ or $w=\{\alpha+n\}$, and $y \subset \alpha+n$. By (3), then

(9) $y \in \mathrm{L}$.

We also have that

(10) $\phi \in L$.

But, since $\alpha+n$ is an ordinal number, hence constructible, and by 9.92 of [8], we have

(11) $\{\alpha+n\} \in \mathrm{L}$.

By (10) and (11),

(12) $w \in \mathrm{L}$.

But, by (9), (12), (8), and 9.85 of [8],

(13) $x \in \mathrm{L}$.

Hence we have shown that $\boldsymbol{p}(\alpha+n+1) \subset \mathrm{L}$, which completes the induction.

We do not know at this time whether this theorem can be strengthened to any great degree. We conjecture that $\boldsymbol{P}(\alpha) \subset \mathrm{L}$ implies that $(\overline{\bar{\beta}}=\vec{\alpha} \rightarrow \boldsymbol{P}(\beta)$ $\subset \mathrm{L})$. If this turns out not to be true, then it would be interesting to see exactly what conditions are necessary and sufficient to have $\boldsymbol{P}(\alpha) \subset \mathbf{L} \rightarrow \boldsymbol{P}(\beta)$ $\subset \mathrm{L}$ for $\dot{\alpha}<\beta, \overline{\bar{\alpha}}=\overline{\bar{\beta}}$.

We do have some information, however, about the cases where $\alpha$ and $\beta$ are distinct initial ordinals. We first present a theorem about regular cardinals. (A cardinal $\aleph_{\beta}$ is regular if there is no increasing sequence of length $<\omega_{\beta}$ of sets of cardinality $<\aleph_{\beta}$ whose limit is a set of cardinality $\aleph_{\beta}$. In particular, if $\beta$ is a successor ordinal, $\aleph_{\beta}$ is regular.)

Theorem II.3 Let $\aleph_{\beta}$ be a regular cardinal. Then $\left(\forall \alpha\left(\alpha<\beta \rightarrow \boldsymbol{P}\left(\omega_{\alpha}\right) \subset \mathrm{L}\right)\right)$ does not imply $\boldsymbol{p}\left(\omega_{\beta}\right) \subset \mathrm{L}$.

Proof: We will use the same methods as Chapter I and attempt to find a model $\mathfrak{R}$ in which $\sim\left(\boldsymbol{P}\left(\omega_{\beta}\right) \subset \mathrm{L}\right)$, but in which $\alpha<\beta \rightarrow \boldsymbol{P}\left(\omega_{\alpha}\right) \subset \mathrm{L}$. We will treat two cases:

(i) $\beta$ is a successor ordinal

(ii) $\beta$ is a limit ordinal. 
Note that, in case (ii), we are saying that $\aleph_{\beta}$ is a weakly inaccessible cardinal. Hence, in this case, we will have to assume that our ground model $\mathfrak{M}$ both satisfies $V=L$ and also allows the existence of a weakly inaccessible cardinal. We also note that, since $V=L$ holds in $\mathfrak{M}$, weak inaccessibility is the same as inaccessibility. (We say that a cardinal number $\aleph_{\beta}$ is inaccessible if $\aleph_{0}<\aleph_{\beta}, \aleph_{\alpha}<\aleph_{\beta} \rightarrow 2^{\aleph_{\alpha}}<\aleph_{\beta}$, and $\aleph_{\beta}$ is regular.)

We now present the proof for (i). Suppose $\beta=\alpha+1$. By Theorem II.1, it suffices to find a model $\mathfrak{N}$ in which $\boldsymbol{P}\left(\omega_{\alpha}\right) \subset L$ but $\sim\left(\boldsymbol{P}\left(\omega_{\alpha+1}\right) \subset L\right)$. As in the proofs in Chapter I, we first define our set $C$ of forcing conditions.

Definition II.3.1 Let $C$ be the set of all sets $p$ of statements of the form " $\delta \epsilon a$ " or " $\sim(\delta \epsilon a)$ " such that $\delta<\omega_{\alpha+1}, \bar{p} \leqq \aleph_{\alpha}$, and for no $\delta$ do we have both " $\delta \epsilon a$ " and " $\sim(\delta \in a)$ " in $p$.

Definition II.3.2 For $p \epsilon \mathrm{C}, p \Vdash \delta \epsilon \mathrm{a}$ if and only if " $\delta \epsilon \mathrm{a}$ " is in $p$.

Definition II.3.3 Let $\left\{p_{n}\right\}_{n<\omega}$ be a complete sequence of forcing conditions. Then $\mathbf{a}=\left\{\delta<\omega_{\alpha+1} \mid \exists k\right.$ such that $\left.p_{k} \Vdash \delta \epsilon a\right\}$.

Lemma II.3.4 $\boldsymbol{P}\left(\omega_{\alpha}\right) \subset$ L.

Proof: By the Solovay lemma (see Lemma I.5.4), we need only show that any chain of conditions of length at most $\omega_{\alpha}$ has an upper bound that is a condition. Suppose we have a sequence of conditions $\left\{p_{\gamma}\right\}_{\gamma<\omega_{\alpha}}$ such that

(1) $\zeta<\eta \rightarrow p_{\zeta} \subset p_{\eta}$.

We now let

(2) $p=\bigcup_{\gamma<\omega_{\alpha}} p_{\gamma}$.

Clearly, $p$ will be a set of statements of the form " $\delta \epsilon a$ " or " $\sim(\delta \epsilon a)$ " and, by (1), $p$ will not contain contradictory statements. Likewise, by (2), $p$ is an upper bound for the $p_{\gamma}$. Therefore, we will have $p \in C$ and our lemma, if we can show that $\overline{\bar{p}} \leqq \aleph_{\alpha^{*}}$. In order to prove this, we note that, by (2), we have

(3) $\overline{\bar{p}}=\overline{\overline{\bigcup_{\gamma<\omega_{\alpha}} p_{\gamma}}}$

But this gives us

(4) $\overline{\bar{p}} \leqq \sum_{\gamma<\omega_{\alpha}} \overline{\overline{p_{\gamma}}}$.

But, since each $p_{\gamma} \in C$ and, hence $\overline{\bar{p}} \leqq \aleph_{\alpha}$, and since there are $\aleph_{\alpha}$ in terms in the summation, we have

(5) $\overline{\bar{p}} \leqq \aleph_{\alpha} \cdot \aleph_{\alpha}$.

But $\aleph_{\alpha} \cdot \aleph_{\alpha}=\aleph_{\alpha}$, hence we have

(6) $\overline{\bar{p}} \leqq \aleph_{\alpha}$,

which is what we wanted to prove. 
Lemma II.3.5 $\sim\left(\boldsymbol{P}\left(\omega_{\alpha+1}\right) \subset \mathrm{L}\right)$.

Proof: It suffices to show that a is not constructible. As in the proof of Lemma I.5.6, we need only show that a is not standard. To do this it is sufficient to prove that, for any $t \in \mathfrak{M}$,

(1) $p \in C \rightarrow p \Vdash \sim(a=t)$.

By condition (a) (for forcing of the negation) which is given in the Introduction, we must show that for no $q$ such that $p \subset q$ do we have $q \Vdash a=\mathbf{t}$. Now suppose we have

(2) $p \in C$

and

(3) $p \subset q \wedge q \in C$.

Since $q \in \mathrm{C}$, we have, by Definition II.3.1,

(4) $\overline{\bar{q}} \leqq \aleph_{\alpha}$.

Therefore, there is some $\zeta<\omega_{\alpha+1}$ such that neither " $\zeta \epsilon a$ " nor " $\sim(\zeta \epsilon a)$ " is in $q$. Now we define $q^{\prime}$ and $q^{\prime \prime}$ as follows:

(5) If $\zeta \in t$, let $q^{\prime}=q \cup\left\{{ }^{\prime} \sim(\zeta \in \mathrm{a})\right.$ ', $\}$.

(6) If $\sim(\zeta \in t)$, let $q^{\prime \prime}=q \cup\{$ “ $\zeta \in \mathrm{a}$ ', $\}$.

Hence, if $\zeta \epsilon t$, by (5), we have

(7) $q^{\prime} \Vdash \zeta \in t \wedge \sim(\zeta \in \mathbf{a})$.

If $\sim(\zeta \in t)$, by $(6)$, we have

(8) $q^{\prime \prime} \Vdash \sim(\zeta \in \mathbf{t}) \wedge \bar{\zeta} \in \mathbf{a}$.

Since we must have either $\zeta \epsilon t$ or $\sim(\zeta \epsilon t)$, either (7) or (8) will hold. Hence, $q$ does not force $\mathbf{a}=\mathbf{t}$. Therefore, $p \Vdash \sim(a=t)$, and $\mathbf{a}$ is not standard. This completes the proof of Lemma II.3.5.

By Lemma II.3.4 and Lemma II.3.5, we have completed the proof of case (i) of Theorem $\Pi$.3. We now prove case (ii).

(ii) If we have a countable model $\mathbb{M}$ in which $V=L$ and $\aleph_{\beta}$ is an inaccessible cardinal (i.e., $\aleph_{\beta}$ is regular and $\beta$ is a limit ordinal), then there is a model $\mathfrak{N}$ in which $\sim\left(\boldsymbol{P}\left(\omega_{\beta}\right) \subset \mathrm{L}\right)$ but $\forall \zeta\left(\zeta<\beta \rightarrow \boldsymbol{P}\left(\omega_{\zeta}\right) \subset \mathrm{L}\right)$.

We proceed as before by first defining $C$, our set of conditions.

Definition II.3.6 For every $\alpha<\beta$, let $C_{\alpha}$ be the set of all sets $p$ of statements of the form " $\delta \epsilon a$ " or " $\sim(\delta \in a)$ " such that $\delta<\omega_{\alpha+1}$ and $\bar{p} \leqq \aleph_{\alpha}$ and such that for no $\delta$ do we have both " $\delta \epsilon a$ ", and " $\sim(\delta \epsilon a)$ "' in $p$. Then: $C=\bigcup_{\alpha<\beta} C_{\alpha}$.

Definition II.3.7 For $p \epsilon C, p \Vdash \delta \epsilon \mathrm{a}$ if and only if " $\delta \epsilon \mathrm{a}$ "' is in $p$.

Definition II.3.8 Let $\left\{p_{n}\right\}_{n<\omega}$ be a complete sequence of forcing conditions. Then $\mathbf{a}=\left\{\delta \mid \delta<\omega_{\beta} . \wedge . \exists k\right.$ such that $\left.p_{k} \Vdash \delta \epsilon \mathrm{a}\right\}$.

Lemma II.3.9 $\forall \zeta\left(\zeta<\beta \rightarrow \boldsymbol{p}\left(\omega_{\zeta}\right) \subset L\right)$. 
Proof: We will again apply Lemma I.5.4, where $\lambda=\omega_{\zeta}$. Let $\left\{p_{\gamma}\right\}_{\gamma<\omega_{\zeta}}$ be a chain of conditions which is of length at most $\omega_{\zeta}$. Now let

(1) $p=\bigcup_{\gamma<\omega_{\zeta}} p_{\gamma}$.

Therefore, we have

(2) $\overline{\bar{p}}=\overline{\bigcup_{\gamma<\omega_{\zeta}} p_{\gamma}}$

and, hence,

(3) $\overline{\bar{p}} \leqq \sum_{\gamma<\omega_{\zeta}} \overline{\bar{p}}_{\gamma}$.

But, by Definition II.3.6, $\overline{\bar{p}}_{\gamma}<\omega_{\beta}$. Therefore, since $\aleph_{\beta}$ is inaccessible, $\omega_{\zeta}<\omega_{\beta}$, and $\bar{p}_{\gamma}<\omega_{\beta}$, we have

(4) $\overline{\bar{p}}<\aleph_{\beta}$.

By (4), and the fact that $\aleph_{\beta}$ is inaccessible, there is some $\mu<\beta$ such that

(5) (" $\alpha \in a$ " in $p$ or " $~(\alpha \in a)$ " in $p) \rightarrow \alpha<\omega_{\mu}$;

otherwise we would have, where " $\alpha \in p$ " means that $\alpha$ occurs in a statement of $p$,

(6) $\bigcup_{\alpha \in p} \alpha=\aleph_{\beta}$

which contradicts the regularity of $\aleph_{\beta}$. Hence, by (4) and (6), if $\overline{\bar{p}}=\aleph_{\nu}$, we have

(7) $p \in C_{\eta}$

where $\eta=\max (\mu, \nu)$. Hence $p \in C$ and the lemma is proved.

Lemma II.3.10 $\sim\left(\boldsymbol{P}\left(\omega_{\beta}\right) \subset L\right)$.

Proof: As before, we need only show that $a$ is not standard. Let $t \in \mathfrak{M}$. It suffices to show that

(1) $p \in C \rightarrow p \Vdash \sim(\mathbf{a}=\mathbf{t})$.

Now suppose we have

(2) $p \in C$

and

(3) $p \subset q \wedge q \in \mathrm{C}$.

Since $q \in C$, by Definition II.3.6,

(4) $\exists \alpha\left(\alpha<\beta . \wedge . q \in C_{\alpha}\right)$.

By (4) and Definition II.3.6, we have

(5) $\delta<\omega_{\alpha} \rightarrow$ neither " $\delta \epsilon a$ " nor " $\sim(\delta \in \mathrm{a})$ ", are in $q$.

We now choose some $\delta$ such that $\omega_{\alpha}<\delta<\omega_{\beta}$, and define $q^{\prime}$ and $q^{\prime \prime}$ as follows:

(6) If $\delta \epsilon t$, let $q^{\prime}=q \cup\left\{\sigma_{\sim}(\delta \in \mathrm{a})\right.$,' $\}$. 
(7) If $\sim(\delta \epsilon t)$, let $q^{\prime \prime}=q \cup\left\{\right.$ " $\left.\delta \epsilon \mathrm{a}^{\prime \prime}\right\}$.

Therefore, if $\delta \in t$, we have, by (6),

(8) $q^{\prime} \Vdash \delta \epsilon t \wedge \sim(\delta \in \mathbf{a})$.

If $\sim(\delta \in t)$, we have, by (8),

(9) $q^{\prime \prime} \Vdash \sim(\delta \in t) \wedge \delta \in \mathbf{a}$.

In either case, $q$ does not force $\mathbf{a}=\mathbf{t}$. Therefore $p \Vdash \sim(\mathbf{a}=\mathbf{t})$, and $\mathbf{a}$ is not standard. This completes the proof of our lemma.

By Lemma II.3.9 and Lemma II.3.10, case (ii) is proved, and, hence, we have completed the proof of Theorem II.3. The reader might ask why we insisted that $\aleph_{\beta}$ be regular. The above proof does not go through for singular cardinals (i.e., cardinals that are not regular). The simplest way to show that this is true is to note that if we have $\aleph_{\beta}$ a singular cardinal and construct the $\mathrm{C}_{\alpha}$ and $C$ as in Definition II.3.6, there is no guarantee that the union of a chain of conditions will be a condition. By weakening Theorem II.3, however, we can obtain a result that holds for singular cardinals as well.

Corollary II.3.11 Let $\aleph_{\beta}$ be a singular cardinal. Then, for every $\alpha$ such that $\alpha<\beta$, there is a model $\mathfrak{N}$ in which $\boldsymbol{P}\left(\omega_{\alpha}\right) \subset \mathrm{L}$ but in which it is not true that $\boldsymbol{p}\left(\omega_{\beta}\right) \subset \mathrm{L}$.

Proof: Since $\aleph_{\beta}$ is singular, $\beta$ is a limit ordinal, Hence, $\alpha<\beta \rightarrow$ there is some ordinal number $\gamma$ such that

(1) $\alpha<\gamma<\beta$

and

(2) $\aleph_{y}$ is regular.

By (1), we have

(3) $\aleph_{\alpha}<\aleph_{\gamma}<\aleph_{\beta}$.

Therefore, by Theorem II.3,

(4) there is a model $\mathfrak{N}$ in which $\boldsymbol{P}\left(\omega_{\alpha}\right) \subset \mathrm{L}$ but $\sim\left(\boldsymbol{P}\left(\omega_{\gamma}\right) \subset \mathrm{L}\right)$.

But, by Theorem II.1, and (1),

(5) $\left(\sim\left(\boldsymbol{P}\left(\omega_{\gamma}\right) \subset L\right) \rightarrow \sim\left(\boldsymbol{P}\left(\omega_{\beta}\right) \subset L\right)\right)$.

Hence, $\mathfrak{R}$ satisfies the desired conditions.

We also have a result that relates these $\boldsymbol{P}\left(\omega_{\alpha}\right) \subset L$ to $V=L$. The proof of this following corollary follows very easily from Theorem II.3 and Corollary II.3.11.

Corollary II.3.12 $\forall \alpha\left(\sim\left(\boldsymbol{P}\left(\omega_{\alpha}\right) \subset \mathrm{L} \rightarrow \mathrm{V}=\mathrm{L}\right)\right)$.

This sharply contrasts with the following result of Lévy [9]. 
Theorem II.4 If the axiom of regularity holds and there is a non-constructible set, then there is a non-constructible set of ordinals.

Changing this to our notation and using the contrapositive, this becomes

Theorem II.4* If we have the axiom of regularity, then $\left(\forall \alpha\left(\boldsymbol{P}\left(\omega_{a}\right) \subset L\right)\right) \rightarrow \vee$ $=\mathrm{L}$.

Thus we see that we can have all the subsets of any ordinal number that we want to be constructible and yet not have $V=L$. But if we choose to have the power set of every ordinal contained in $L$, then we must have $V=L$. We will treat Theorem II.4* more thoroughly in Chapter III.

We now look at the effect of the $\boldsymbol{P}\left(\omega_{a}\right) \subset \mathrm{L}$ on the axiom of choice.

Theorem II.5 $\forall \beta\left(\sim\left(\boldsymbol{P}\left(\omega_{\beta}\right) \subset \mathrm{L} \rightarrow \mathbf{A C}\right)\right)$.

We will proceed as in earlier proofs.

Definition II.5.1 Let $C$ be the set of all sets $p$ of statements of the form " $\alpha \epsilon \mathrm{a}_{\delta}$ " or " $~\left(\alpha \in \mathrm{a}_{\delta}\right)$ " for $\alpha<\omega_{\beta+1}, \delta<\omega_{\beta+1}$ such that not both " $\alpha \epsilon \mathrm{a}_{\delta}$ " and " $\sim\left(\alpha \in \mathrm{a}_{\delta}\right)$ ", occur in $p$ for any $\alpha$ or $\delta$, and such that $\bar{p} \leqq \aleph_{\beta}$.

We now introduce to our language a new constant $W$.

Definition II.5.2 For $p \in C, \quad p \Vdash \alpha \epsilon \mathrm{a}_{\delta} \leftrightarrow$ " $\alpha \epsilon \mathrm{a}_{\delta}$ " $\epsilon p . \quad p \Vdash \mathrm{a}_{\delta} \in \mathrm{W}$ for every $p \in C$ and for every $\delta<\omega_{\beta+1}$.

Definition II.5.3 Let $\left\{p_{n}\right\}_{n<\omega}$ be a complete sequence of forcing conditions. For each $\delta<\omega_{\beta+1}, a_{\delta}=\left\{\alpha \mid \exists k\right.$ such that $\left.p_{k} \Vdash \alpha \epsilon a_{\delta}\right\} ; \mathbf{W}=\left\{\boldsymbol{a}_{\delta} \mid \exists k\right.$ such that $\left.p_{k} \Vdash a_{\delta} \in W\right\}$.

Hence, by Definitions II.5.2 and II.5.3, we have $\mathbf{W}=\left\{\mathbf{a}_{\delta} \mid \delta<\omega_{\beta+1}\right\}$.

Lemma II.5.4 $\boldsymbol{P}\left(\omega_{\beta}\right) \subset \mathrm{L}$ is true in $\mathfrak{N}$.

Proof: This follows by exactly the same proof as that of Lemma II.3.4.

Definition II.5.5 Let $g$ be the group of all permutations $\pi$ of $\omega_{\beta+1}$ such that $\pi(\alpha)=\alpha$ for all $\alpha<\omega_{\beta+1}$ except for a set $x$ of ordinal numbers such that $\overline{\bar{x}} \leqq \aleph_{\beta}$. Let $g_{\alpha}$ be the subgroup of $g$ defined by: $g_{\alpha}=\{\pi \mid \pi(\gamma)=\gamma$ for all $\gamma \leqq \alpha\}$.

If $\pi$ is a permutation in $g$, we extend to a permutation on $\mathfrak{N}$ in the following manner:

Definition II.5.6

(i) $\pi(\mathbf{t})=\mathbf{t}$ for $\pi \in \boldsymbol{g}, t \in \mathfrak{R}$.

(ii) $\pi\left(\mathbf{a}_{\delta}\right)=\mathbf{a}_{\pi(\delta)}$ for $\pi \epsilon g, \delta<\omega_{\beta+1}$.

(iii) $\pi(W)=W$ for all $\pi \in g$.

(iv) For $t \in \mathfrak{R}, \pi \in \mathfrak{O}$, if $t$ corresponds to the formula $\mathfrak{A} \leftrightarrow \mathfrak{B}\left(t_{1}, \ldots, t_{n}\right)$ where $t_{i} \in \mathfrak{R}$, then $\pi(\mathfrak{A}) \leftrightarrow \mathfrak{B}\left(\pi\left(t_{1}\right), \ldots, \pi\left(t_{n}\right)\right)$.

(v) If $p$ is a forcing condition, $\pi(p)$ is the forcing condition defined by: " $\alpha \epsilon \mathrm{a}_{\delta}$ " $\epsilon p \leftrightarrow$ " $\alpha \epsilon \mathrm{a}_{\pi(\delta)}$ ", $\epsilon \pi(p)$ and " $\sim\left(\alpha \in \mathrm{a}_{\delta}\right)$ ", $\epsilon p \leftrightarrow$ " $\sim\left(\alpha \in \mathrm{a}_{\pi(\delta)}\right)$ "' $\epsilon \pi(p)$.

Lemma II.5.7 $p \subset q \leftrightarrow \pi(p) \subset \pi(q)$. 
Proof: This follows from Definition II.5.6 (v).

Lemma ח.5.8 $p \Vdash \mathfrak{A} \leftrightarrow \pi(p) \Vdash \pi(\mathfrak{A})$.

Proof: This proof is exactly the same as the proof of Lemma I.7.8.

Lemma II.5.9 For each $t \in \mathfrak{N}$, formula $\mathfrak{A}$, and $p \in \mathrm{C}$, there is an $\alpha<\omega_{\beta+1}$ such that $\pi \in \mathscr{g}_{\alpha} \rightarrow \pi(t)=t, \pi(\mathfrak{A})=\mathfrak{A}$, and $\pi(p)=p$.

Proof: Same as for Lemma I.7.9.

Lemma II.5.10 In $\mathfrak{N}, \mathbf{W}$ is a subset of $\boldsymbol{p}\left(\omega_{\beta+1}\right)$ such that $\sim\left(\overline{\overline{\mathbf{W}}}<\boldsymbol{\aleph}_{\beta+1}\right)$ and yet $\mathbf{W}$ does not contain a subset of cardinality $\aleph_{\beta+1}$.

Proof: As in the proof of Lemma I.6.5, we have, for any $p \in C$,

(1) $\sim(\gamma=\delta) \rightarrow p \Vdash \sim\left(\mathbf{a}_{\gamma}=\mathbf{a}_{\delta}\right)$.

Since, in $\mathfrak{R}$, each $\boldsymbol{a}_{\delta} \in \mathbf{W}$, we have

(2) $\sim\left(\overline{\overline{\mathbf{W}}}<\aleph_{\beta+1}\right)$,

since we have specified $\aleph_{\beta+1}$ elements of $\mathbf{W}$. Now let $t \in \mathfrak{N}$ and assume that for some $p$ in $\left\{p_{n}\right\}_{n<\omega}$

(3) $p \Vdash\left(t\right.$ is a one-one function from $\omega_{\beta+1}$ into $\left.W\right)$.

Let $\zeta$ be an ordinal number such that $\zeta<\omega_{\beta+1}$ and let $g_{\zeta}$ be defined as in Definition II.5.5. Also suppose that $\zeta$ satisfies

(4) $\pi \in g_{\zeta} \rightarrow \pi(t)=t$

and that $\zeta$ is greater than any $\delta$ such that $a_{\delta}$ occurs in a statement of $p$. Since $t$ must take on $\aleph_{\beta+1}$ distinct values, there must be some $p^{\prime}$ in the complete sequence $\left\{p_{n}\right\}_{n<\omega}$ such that $p \subset p^{\prime}$ and

(5) $p^{\prime} \Vdash t(\eta)=\mathbf{a}_{\sigma}$

for some $\eta$ and $\sigma$ where $\sigma$ is greater than $\zeta$. Let $\tau>\zeta$ be an ordinal number such that $\tau<\omega_{\beta+1}$ and $a_{\tau}$ does not appear in $p^{\prime}$; let $\pi$ be the permutation which interchanges $\sigma$ and $\tau$ and is the identity on all other ordinals $<\omega_{\beta+1}$. If $p^{\prime \prime}=\pi\left(p^{\prime}\right)$, then, by (5),

(6) $p^{\prime \prime} \Vdash t(\eta)=\mathbf{a}_{\tau}$.

We also have that, if $q=p^{\prime} \cup p^{\prime \prime}$, then

(7) $q$ is a forcing condition.

Since $p \subset q$, and by (3), $q$ forces $t$ to be a function. But, by (5), we have, since $p^{\prime} \subset q$,

(8) $q \Vdash t(\eta)=\mathbf{a}_{\sigma}$.

But, by (6), $p^{\prime \prime} \subset q$, therefore,

(9) $q \Vdash t(\eta)=\mathbf{a}_{\tau}$.

Hence, (3), (8), and (9) give a contradiction and our lemma is proved. 
By Lemma II.5.10, we see that $\sim\left(\aleph_{\beta+1} \leqq \overline{\overline{\mathbf{W}}}\right)$ and also that $\sim\left(\overline{\overline{\mathbf{W}}}<\aleph_{\beta+1}\right)$. Hence, we do not have trichotomy for cardinal numbers and $A C$ fails. By Lemma II.5.4 and Lemma II.5.10, we have the proof of Theorem II.5.

We have now seen the effects of the propositions $\boldsymbol{P}\left(\omega_{\alpha}\right) \subset L$ on $V=L$ and AC. We shall now show their effect on $G C H$. Since $G C H$ implies $A C$, and by Theorem II.5, we immediately have:

Theorem II.6 $\forall \beta\left(\sim\left(\mathcal{P}\left(\omega_{\beta}\right) \subset \mathbf{L} \rightarrow \mathbf{G C H}\right)\right)$.

We can, however, strengthen this result to show the various ways in which GCH can be violated while still assuming $\boldsymbol{P}\left(\omega_{\zeta}\right) \subset \mathrm{L}$. Using the results of Easton's doctoral dissertation, [5], we will prove that if we assume $\boldsymbol{P}\left(\omega_{\zeta}\right) \subset \mathrm{L}$ for some initial ordinal $\omega_{\zeta}$, then for any initial ordinal number $\omega_{\alpha}>\omega_{\zeta}$ we can assume $2^{\aleph \alpha}$ to be any cardinal that is compatible with König's theorem. Because of the addition of $\boldsymbol{P}\left(\omega_{\zeta}\right) \subset L$, our theorem will be a slight strengthening of Easton's. We will, however, have to assume that $V=L$ holds in our ground model (or at least that $\mathrm{GCH}+\boldsymbol{P}\left(\omega_{\zeta}\right) \subset \mathrm{L}$ holds in the ground model) instead of simply $\mathbf{G C H}$ as Easton does. For this reason our theorem is in some respects weaker than Easton's.

We will pattern our proof very closely after Easton's; hence, we will use the notation and numbering of [5]. For this reason, we will use the Gödel-Bernays axiomatization of set theory (including $A C$ ) instead of $Z F+A C$. This Gödel-Bernays set theory $+A C$ will be denoted $\Sigma_{*}$. Most of the definitions and lemmas in this proof will be taken directly from [5]. For the sake of completeness, we shall state all of the pertinent lemmas and definitions as they occur in [5]. If no change in the proof of a lemma is desired or needed, we will simply state the lemma without proof, but in smaller type. Any change from [5] will be pointed out at its occurrence, but we will have to remember that the form of some of our lemmas might be the same as Easton's although the meaning is different due to the change of some definitions. For ease of reference, in this part of Chapter II we will use the same enumeration as in [5].

We now state Easton's main theorem.

Theorem (Easton) Let $\mathfrak{M}$ be a countable model of $\Sigma_{*}$ in which $\mathrm{GCH}$ holds and let $G$ be a function in $\mathfrak{M}$ such that:

(i) $\alpha \leqq \beta$ implies $G_{\alpha}^{\prime} \leqq G_{\beta}^{\prime}$.

(ii) $\aleph_{G_{\alpha}^{\prime}}$ is not cofinal with any cardinal less than or equal to $\aleph_{\alpha}$.

Then there is an extension $\mathfrak{N}$ of $\mathfrak{M}$ in which cardinals are absolute and $2^{\aleph_{\alpha}}=$ $\aleph_{G_{\alpha}^{\prime}}$ for regular cardinals $\aleph_{\alpha}$.

We say that cardinals are absolute in the extension if $\mathfrak{M}$ and $\mathfrak{R}$ have the same cardinals and $\aleph_{\alpha}<\aleph_{\beta}$ in $\mathfrak{M}$ if and only if $\aleph_{\alpha}<\aleph_{\beta}$ in $\mathfrak{N}$. We now modify this to the following:

Theorem II.7 Let $\mathfrak{M}$ be a countable model of $\Sigma_{*}$ in which $V=L$ holds; let $\zeta$ be any ordinal number, and let $G$ be a function in $\mathfrak{M}$ defined on ordinal numbers larger than $\zeta$ such that: 
(i) $\zeta<\alpha \leqq \beta$ implies $G^{\prime} \alpha \leqq G^{\prime} \beta$.

(ii) $\aleph_{G^{\prime} \alpha}$ is not cofinal with any cardinal less than or equal to $\aleph_{\alpha}$.

Then there is an extension $\mathfrak{R}$ of $\mathfrak{M}$ in which cardinals are absolute, $\boldsymbol{P}\left(\omega_{\zeta}\right) \subset \mathrm{L}$ and $2^{\aleph_{\alpha}}=\aleph_{G_{\alpha}^{\prime}}$ for regular cardinals $\aleph_{\alpha}$ such that $\aleph_{\zeta}<\aleph_{\alpha}$.

Proof: We will follow the proof of Easton's theorem. Easton defines the relation $\mathrm{A}(\gamma, \alpha, \eta)$ to be the same as $\gamma \in \mathrm{a}_{\eta}^{\alpha}$. We will have a language $\mathcal{\alpha}$, which has functional constants $\epsilon$ and $A$. There will be ranked variables $v_{i}^{\alpha}$ for each ordinal $\alpha$ and each integer $i$ of $\mathfrak{M}$ and there will be variables $v_{i}$ for each integer of $\mathfrak{M}$.

Definition 1 We give an inductive definition of ranked formula and of abstraction term. The definition is to be given in the model $\mathfrak{m}$.

1. If $u, v$ and $w$ are abstraction terms, set constants, or ranked variables, then $\mathrm{A}(u, v, w)$ and $u \epsilon v$ are ranked formulas.

2. If $\Phi$ and $\Psi$ are ranked formulas, then $\sim \Phi, \Phi \vee \Psi$ and $\left(\exists x^{\alpha}\right) \Phi$ are ranked formulas.

3. If $\Phi$ is ranked formula containing no free variables other than $x^{\alpha}$, no occurrences of $\left(\exists y^{\beta}\right) \Psi$ with $\beta>\alpha$, and no occurrences of abstraction terms $\hat{y}^{\beta} \Psi$ or set constants of rank $\beta$ with $\beta \geqq \alpha$, then $\hat{x}^{\alpha} \Phi$ is an abstraction term. (Where $\hat{x} \Phi=$ the class of all $x$ such that $\Phi(x) ; \hat{y}^{\alpha} \Phi=$ the class of all $y^{\alpha}$ such that $\Phi\left(y^{\alpha}\right)$.)

Definition 2 The rank of a variable, abstraction term, or set constant is given by:

1. $\rho\left(x^{\alpha}\right)=\alpha$

2. $\rho\left(\hat{x}^{\alpha} \Phi\right)=\alpha$

3. $\rho(s)$ is the rank of the set $s$.

Definition 3 We now define (unranked) formulas of the language $\mathcal{L}$. The definition is to take place in the metalanguage rather than in $\mathfrak{M}$.

1. If $u, v$ and $w$ are variables or constant terms, then $u \epsilon v$ and $\mathrm{A}(u, v, w)$ are formulas. Furthermore, if $S$ is any constant, then $u \in S$ is a formula.

2. If $\Phi$ and $\Psi$ are formulas, then $\sim \Phi, \Phi \vee \Psi,(\exists x) \Phi$, and $\left(\exists x^{\alpha}\right) \Phi$ are formulas.

Lemma 1 There is an assignment of sets of $\mathfrak{M}$ to ranked formulas of $\{$ such that the collection of sets assigned to ranked formulas is a class of the model $\mathfrak{M}$ and the usual syntactical operations (forming negations, substitution, etc.) are represented by functions of $\mathbb{M}$.

Easton introduces the equality relation (for unranked formulas) by the following definition:

$$
u=v \rightarrow \forall x(x \in u \leftrightarrow x \in v) .
$$

In addition he introduces the expression $u \cong v$, where $u$ and $v$ are constant terms, by

$$
u \cong v \rightarrow \forall x^{\gamma}\left(x^{\gamma} \epsilon u \leftrightarrow x^{\gamma} \epsilon v\right),
$$

where $\gamma=\max (\rho(u), \rho(v))$.

In the following definition we make the most significant deviation from Easton's method in order to achieve the desired result. Note that we use the class $\operatorname{Reg}_{\zeta}$ instead of the class Reg which Easton uses (where Reg is the class of all ordinals $\alpha$ such that $\aleph_{\alpha}$ is regular).

Definition $4 A$ set of conditions is a set $q$ of the model $\mathfrak{M}$ of quadruples $\langle 0 \gamma a \eta\rangle$ and $\langle 1$ r $\alpha \eta\rangle$ with the following properties:

(i) $q=\bigcup_{\alpha \in \operatorname{Reg} \zeta} q^{\alpha}$, where $\operatorname{Reg}_{\zeta}$ is the class of ordinals $\alpha$ such that $\aleph_{\alpha}$ is regular and $\zeta<\alpha$, and 
(ii) $q^{\alpha}$ is a set of quadruples $\langle i \gamma \alpha \eta\rangle, i<2, \gamma<\aleph_{\alpha}$, and $\eta<\aleph_{G^{\prime} \alpha}$.

(iii) For $\alpha \in \operatorname{Reg}_{\zeta}, \bigcup_{\beta \leq \alpha} q^{\beta}$ is of cardinality $<\aleph_{\alpha}$.

(iv) For no $\gamma, \alpha$, and $\eta$, does $q$ contain both $\langle 0 \gamma \alpha \eta\rangle$ and $\langle 1 \gamma \alpha \eta\rangle$.

The following definitions and lemmas (up to but not including Definition 15) are the same as in [5]. However, we must keep in mind that we now have a different class of sets of conditions, due to the use of $\operatorname{Reg}_{\zeta}$ instead of Easton's Reg.

Definition 5 Sc is the class of all sets of conditions.

Definition $6 \quad p^{\prime}$ is an extension of a set of conditions $p$ if $p^{\prime}$ is a set of conditions and $p \subset p^{\prime}$.

Definition 7 For a ranked statement $\Phi$, we set:

$$
\operatorname{ord}(\Phi)=\omega^{2} \cdot \alpha+\omega \cdot t+l
$$

where:

$\alpha$ is the least ordinal such that $\Phi$ contains no variable of rank $>\alpha$ and no constant term of rank $\geqq \alpha$.

$t=0$ if $\Phi$ contains no subformula of the form $v \in u$, where $v$ is a constant term of rank $\alpha$, and no subformula $\mathrm{A}(u, v, w)$ other than inside an abstraction term; otherwise, $t=1$.

$l$ is the length of the formula $\Phi$. (u€v and $\mathrm{A}(u, v, w)$ have length 1.)

Definition 8 The rank of a set of conditions $p$, $(\operatorname{rank}(p))$ is the supremum of the ranks of its elements, where $\langle i \gamma \alpha \eta\rangle$ is said to have $\operatorname{rank} \max (\gamma, \alpha, \eta)$.

We note that with our modifications on the conditions, if $p$ is a set of conditions, then $\operatorname{rank}(p)>\zeta$.

Definition $9 p \Vdash \Phi$ is defined (for ranked $\Phi$ ) in terms of $p^{\prime} \Vdash \Psi$, ord $(\Psi)<\operatorname{ord}(\Phi)$ and $\operatorname{rank}\left(p^{\prime}\right) \leqq \max (\operatorname{ord}(\Phi), \operatorname{rank}(p))$, as follows:

1. $p \Vdash \sim \Phi$ if there is no set of conditions $p^{\prime}, \operatorname{rank}\left(p^{\prime}\right) \leqq \operatorname{ord}(\Phi)$, such that $p^{\prime}$ is compatible with $p$ and $p^{\prime} \Vdash \Phi$. (Sets of conditions $p$ and $p^{\prime}$ are said to be compatible if their union $p \cup p^{\prime}$ is a set of conditions.)

2. $p \Vdash \Phi \vee \Psi$ if either $p \Vdash \Phi$ or $p \Vdash \Psi$ (or both).

3. $p \Vdash\left(\exists x^{\alpha}\right) \Phi\left(x^{\alpha}\right)$ if $p \Vdash \Phi(u)$ for some constant term $u, \rho(u)<\alpha$.

4. $p \Vdash u \in \mathrm{s}$ if $p \Vdash u \cong \mathbf{t}$ for some $t \in S$.

5. $p \Vdash u \in \hat{x} \Phi(x)$ if for some constant term $u^{\prime}, \rho\left(u^{\prime}\right)<\alpha, p \Vdash u \cong u^{\prime}$, and $p \Vdash \Phi\left(u^{\prime}\right)$.

6. $p \Vdash A(u, v, w)$ if there exist ordinals $\gamma, \alpha$, and $\eta$, such that $\gamma \leqq \rho(u), \alpha \leqq \rho(v)$, $\eta \leqq \rho(w), p \Vdash u \cong \gamma, p \Vdash v \cong \alpha, p \Vdash w \cong \eta$, and $p$ contains $\langle 0 \gamma \alpha \eta\rangle$.

7. $p \Vdash \Phi$ only as required by 1-6 above.

Definition $10 p \Vdash \Phi$ is defined for unranked $\Phi$ by induction on the length of the statement $\Phi$.

1. $p \Vdash u \epsilon v, p \Vdash \mathrm{A}(u, v, w)$ if so required by Definition 9 .

2. $p \Vdash u \in \mathrm{S}$ if for some $t \in S, p \Vdash u \cong \mathbf{t}$.

3. $p \Vdash \sim \Phi$ if there is no extension $p^{\prime}$ of $p$ such that $p^{\prime} \Vdash \Phi$.

4. $p \Vdash \Phi \vee \Psi$ if $p \Vdash \Phi$ or $p \Vdash \Psi$ (or both).

5. $p \Vdash\left(\exists x^{\alpha}\right) \Phi\left(x^{\alpha}\right)$ if, for some constant term $u, p \Vdash \Phi(u)$.

6. $p \Vdash \Phi$ only as required by $1-5$ above.

Lemma 2 There is a class in the model $\mathfrak{M}$ whose elements are the pairs 〈 $p$ " $\Phi$ "〉 such that $\Phi$ is a ranked statement and $p \Vdash \Phi$. (Where " $\Phi$ " is the set corresponding to the ranked formula $\Phi$ which was defined by Lemma 1.)

Lemma 3 Let $\Phi\left(x_{1}, \ldots, x_{n}\right)$ be an unranked formula of $\mathcal{L}$. There is a class of the model $\mathfrak{M}$ whose elements are the $(n+1)$-tuples $\left\langle p\right.$, ' $u_{1}$ ', $\ldots$, ' $u_{n}$ ' $\rangle$ such that $p \Vdash \Phi\left(u_{1}, \ldots, u_{n}\right)$. 
Lemma 4 If $\Phi$ is a ranked formula and $p \Vdash \Phi$, then $p^{\prime} \Vdash \Phi$ for any extension $p^{\prime}$ of $p$.

Lemma 5. $p \Vdash \sim \Phi \leftrightarrow$ no extension $p^{\prime}$ of $p$ forces $\Phi$.

Lemma 6 No set of conditions forces both a statement $\Phi$ and its negation $\sim \Phi$.

Lemma $7 \quad$ Let $p$ be a set of conditions and let $\Phi$ be a statement of $\mathcal{L}$. Then there is an extension $p^{\prime}$ of $p$ such that either $p^{\prime} \Vdash \Phi$ or $p^{\prime} \Vdash \sim \Phi$.

Lemma 8 Let $p \Vdash \Phi$ and let $p^{\prime}$ be an extension of $p$. Then $p^{\prime} \Vdash \Phi$.

Definition $11 p \Vdash^{*} \Phi, p$ weakly forces $\Phi$, if $p \Vdash \sim \sim$.

Lemma 9 The weak forcing relation has the following properties.

(i) $p \Perp^{*} \Phi \leftrightarrow n o$ extension of $p$ forces $\sim \Phi$.

(ii) $p \Vdash \Phi \rightarrow p \Vdash^{*} \Phi$.

(iii) $p \Vdash^{\bar{*}} \sim \Phi \leftrightarrow p \Vdash \sim \Phi$.

(iv) If $\Phi$ is of the form $\Psi \wedge \Upsilon, \Psi \leftrightarrow \Upsilon, \forall x \Psi, \forall x^{\alpha} \Psi, u \cong v$, or $u=v$, then $p \Vdash \Phi \leftrightarrow p \Vdash^{*} \Phi$.

(v) $p \Vdash^{*} \forall x \Phi(x) \leftrightarrow p \Vdash \Phi(u)$ for all constant terms $u$.

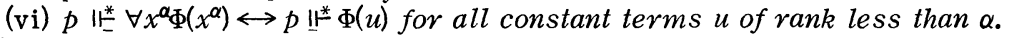

(vii) $p \Perp_{-}^{*} \Phi \leftrightarrow \Psi$ and $p \Perp_{-}^{*} \Phi \rightarrow p \Perp_{-}^{*} \Psi$.

(viii) $p \|^{\bar{*}} \Phi \leftrightarrow \Psi$ and $p \Vdash^{\bar{*}} \Psi \rightarrow p \|^{\bar{*}} \Phi$.

Lemma $10 \quad p \Vdash u \cong v \leftrightarrow p \Vdash u=v$.

Lemma 11 For any set of conditions $p$,

(i) $p \Vdash u=u$.

(ii) $p \Vdash u=v \leftrightarrow p \Vdash v=u$.

(iii) $p \Vdash u=v$ and $p \Vdash v=w \rightarrow p \Vdash u=w$.

Lemma 12 For any set of conditions $p$,

(i) $p \Vdash u \epsilon w$ and $p \Vdash u=v \rightarrow p \Vdash v \epsilon w$.

(ii) $p \Vdash w \epsilon u$ and $p \Vdash u=v \rightarrow p \Vdash * w \in v$.

Lemma 13 If $p \Vdash u=\alpha$, then $\rho(u) \geqq \alpha$.

Corollary 13.1 If $p \Vdash A(u, v, w)$ and $p \Vdash u=u^{\prime}, p \Vdash v=v^{\prime}$, and $p \Vdash w=w^{\prime}$, then $p \Vdash \mathrm{A}\left(u^{\prime}, v^{\prime}, w^{\prime}\right)$.

Definition 12 A sequence of sets of conditions $p^{(0)} \subset p^{(1)} \subset \ldots$ is said to be complete if for every class $C$ of sets of conditions such that every set of conditions has an extension in $C, p^{(k)} \in C$ for some $k$.

Lemma 14 Let $p^{(0)} \subset p^{(1)} \subset \ldots$ be a complete sequence of sets of conditions. Then every statement or its negation is eventually forced by some $p^{(k)}$.

Lemma 15 There exists a complete sequence of sets of conditions.

Definition 13 (Definition of the model $\mathfrak{R}$ )

(i) Let $\Phi(x)$ be an unranked formula of $\&$. The collection of all constant terms $v$ such that for some $k, p^{(k)} \Vdash \Phi(v)$ will be a class of the model $\mathfrak{N}$; we will denote this class by $\hat{x} \Phi(x)$.

(ii) Sets of the model $\mathfrak{N}$ will be classes of the form $\hat{x}(x \in u)$, where $u$ is a constant term of $\mathcal{L}$.

(iii) The $\epsilon$-relation is defined as follows:

$\hat{x} \Phi(x) \in \hat{y} \Psi(y)$ will hold if $\hat{x} \Phi(x)$ is the same as $\hat{x}(x \in u)$ for some constant term $u$ and $p^{(k)} \Vdash \Psi(u)$ for some $k$.

(iv) The relation $\mathrm{A}(\hat{x} \Phi(x), \hat{y} \Psi(y), \hat{x} \Upsilon(z))$ will hold if for some $\gamma, \alpha$, and $\eta$ such that $\langle 0 \gamma \alpha \eta\rangle$ is contained in some $p^{(k)}, \hat{x} \Phi(x)$ is $\hat{x}(x \in \gamma), \hat{y} \Psi(y)$ is $\hat{y}(y \in \alpha)$, and $\hat{z} \Upsilon(z)$ is $\hat{z}(z \in \eta)$.

(v) If $W$ is a constant term or constant of $\mathcal{L}$, then $W$ denotes the class $\hat{x}(x \in W)$.

(vi) Individual variables $x, y, \ldots$, range over all sets of $\mathfrak{N}$; ranked variables of rank $\alpha$ range over sets of the form $\hat{x}(x \in u)$ where $u$ is a constant term of rank less than $\alpha$. 
Definition 14 We will say that the ranked or unranked statement $p$ is eventually forced if for some $k, p^{(k)} \Vdash \Phi$.

Lemma $16 \hat{x} \Phi(x)$ is the same as $\hat{y} \Psi(y)$ if and only if $\forall x(\Phi(x) \leftrightarrow \Psi(x))$ is eventually forced. Hence, if $U$ and $W$ are constants or constant terms, then $U=W$ if and only if $U=W$ is eventually forced.

Lemma 17 An unranked statement $\Phi$ of $\mathcal{L}$ is true in the model $\mathfrak{N}$ if and only if $\Phi$ is eventually forced.

Lemma 18 If $\Phi$ is an unranked statement of $\mathcal{L}$, then $p \Perp^{*} \Phi$ if and only if $\Phi$ is true in all models obtained by the above construction from complete sequences of sets of conditions in which poccurs.

Corollary 18.1 If $\Phi(x)$ is a ranked formula of finite length, then $u \in \hat{x}^{\alpha} \Phi\left(x^{\alpha}\right)$ is true in $\mathfrak{N}$ if and only if $u=u^{\prime}$ for some $u^{\prime}$ of rank less than $\alpha$ and $\Phi(u)$ is true in $\mathfrak{N}$.

Lemma 19 The mapping $\Psi$ of $\mathfrak{M}$ into $\mathfrak{R}$ given by $\Psi(S)=\mathbf{S}$ is an isomorphism with respect to the $\epsilon$-relation.

With Lemmas 20-23, Easton shows that the axioms of $\Sigma *$, except for replacement, power set, and $A C$, hold in the model $\mathfrak{R}$. He now goes on to verify these remaining axioms. In the following definition and its applications we again must use the class Reg $_{\zeta}$ instead of Easton's Reg.

Definition 15 We define $\Gamma_{\alpha}$ and $\Delta_{\alpha}$ as follows:

(i) $\Gamma_{\alpha}=\left\{2 \times \aleph_{\beta} \times\{\beta\} \times \aleph_{G^{\prime} \beta} \mid \beta \in \operatorname{Reg}_{\zeta} \cdot \wedge . \beta \leqq \alpha\right\}$

(ii) $\Delta_{\alpha}=\left\{2 \times \aleph_{\beta} \times\{\beta\} \times \aleph_{G^{\prime}} \mid \beta \in \operatorname{Reg}_{\zeta} \cdot \wedge . \beta>\alpha\right\}$,

where, as in Definition $4, \operatorname{Reg}_{\zeta}$ is the class of ordinal numbers $\alpha$ such that $\aleph_{\alpha}$ is regular and $\zeta<\alpha$.

We note that if $\alpha \leqq \zeta$, then $\Gamma_{\alpha}=\phi$.

Definition 16 Sets of conditions $p$ and $q$ are said to be compatible if their union is again a set of conditions, i.e., if it is not the case that one of them contains a quadruple $\langle 0 \gamma \alpha \eta\rangle$ while the other contains $\langle 1$ r $\alpha \eta\rangle$.

Lemma 24 is stated exactly as in [5]. But because of our use of Reg Re $_{\zeta}$ its proof is somewhat different from that given by Easton. For the same reason we must give the new proofs for the next several lemmas.

Lemma 24 Let $\aleph_{\alpha}$ be a regular cardinal of $\mathfrak{M}$, and let $q$ be a set of conditions, $q \subset \Delta_{\alpha}$, and let $\Phi$ be a statement of $\dot{\mathcal{L}}$. Then there is an extension $\bar{q} \subset \Delta_{\alpha}$ of $q$ and $a$ set $\Pi$ of sets of conditions such that:

(i) $\overline{\bar{\Pi}} \geqq \aleph_{\alpha}$.

(ii) $p \in \Pi \rightarrow$ either $p \cup \bar{q} \Vdash \Phi$ or $p \cup \bar{q} \Vdash \sim \Phi$.

(iii) If $p^{\prime}$ is any set of conditions, there is some $p \in \Pi$ compatible with $p^{\prime}$.

(iv) $p \in \Pi \rightarrow p \subset \Gamma_{\alpha}$.

Proof: If $\alpha>\zeta$, the proof is exactly the same as Easton's. If $\alpha \leqq \zeta$, we note, by Definition 15, that

(1) $\Gamma_{\alpha}=\phi$.

In this case we let

(2) $\Pi=\{\phi\}$.

By (2), it is obviously true that 
(3) (i) and (iv) are satisfied.

By Definition 16, $\phi$ is compatible with any set of conditions, hence, we have

(4) (iii) is satisfied.

By Lemma 7, there is an extension $\bar{q}$ of $q$ such that

(5) $\bar{q} \Vdash \Phi$ or $\bar{q} \Vdash \sim \Phi$.

By (1)

(6) $\bar{q} \subset \Delta_{\alpha}$.

Since $p \in \Pi$ means $p=\phi$, we have

(7) $p \in \Pi \rightarrow p \cup \bar{q}=\bar{q}$.

Hence, by (5), (6) and (7),

(8) (ii) is satisfied.

By (3), (4) and (8), our lemma is proved for $\alpha \leqq \zeta$.

Lemma 25 Let $\beta$ be any ordinal of $\mathfrak{M}$, let $q$ be a set of conditions, let $\Phi\left(x_{1}, \ldots, x_{n}\right)$ be an unranked formula of $\mathcal{L}$, and let $u_{i_{\mu}}, 1 \leqq i \leqq n$ and $\mu<\aleph_{\beta}$ be constant terms. There is an extension $\bar{q}$ of $q$ and $a$ set $\Pi$ of sets of conditions such that:

(i) $\overline{\bar{\Pi}} \leqq \aleph_{\beta}$.

(ii) If $q^{\prime}$ is an extension of $\bar{q}$, and $\mu<\aleph_{\beta}$, there is some $p \in \Pi$ compatible with $q^{\prime}$ such that either $p \cup \bar{q} \Vdash \Phi\left(u_{1_{\mu}}, \ldots, u_{n_{\mu}}\right)$ or $p \cup \bar{q} \Vdash \sim \Phi\left(u_{1_{\mu}}, \ldots, u_{n_{\mu}}\right)$.

(iii) $p \in \Pi \rightarrow p \subset \Gamma_{\beta}$.

Proof: We treat the case where $\beta \leqq \zeta$ in exactly the same way as in the proof of Lemma 24. The proof for $\beta>\zeta$ holds as in [5].

Lemma 26 Let $q$ be a set of conditions and let $\Phi\left(x_{1}^{\gamma}, \ldots, x_{n}^{\gamma}\right)$ be an unranked formula. Then there is an extension $\bar{q}$ of $q$ and an ordinal $\delta$ such that

$$
\bar{q} \Vdash \forall x_{1}^{\gamma} \ldots \forall x_{n}^{\gamma}\left(\exists y \Phi\left(x_{1}^{\gamma}, \ldots, x_{n}^{\gamma}, y\right) \leftrightarrow \exists y^{\delta} \Phi\left(x_{1}^{\gamma}, \ldots, x_{n}^{\gamma}, y^{\delta}\right)\right) .
$$

Corollary 26.1 Let $\Phi\left(x_{1}^{\gamma}, \ldots, x_{n}^{\gamma}, y\right)$ be an unranked formula. Then there is an ordinal $\delta$ such that:

$$
\forall x_{1}^{\gamma} \ldots \forall x_{n}^{\gamma}\left(\exists y \Phi\left(x_{1}^{\gamma}, \ldots, x_{n}^{\gamma}, y\right) \leftrightarrow \exists y^{\delta} \Phi\left(x_{1}^{\gamma}, \ldots, x_{n}^{\gamma}, y^{\delta}\right)\right)
$$

is true in the model $\mathfrak{R}$.

Lemma 27 Let $\Phi\left(x_{1}^{\gamma}, \ldots, x_{n}^{\gamma}\right)$ be an unranked formula. Then there is a formula $\Phi^{\prime}\left(x_{1}^{\gamma}, \ldots, x_{n}^{\gamma}\right)$ which is both a ranked and an unranked formula such that

$$
\forall x_{1}^{\gamma} \ldots \forall x_{n}^{\gamma}\left(\Phi\left(x_{1}^{\gamma}, \ldots, x_{n}^{\gamma}\right) \leftrightarrow \Phi^{\prime}\left(x_{1}^{\gamma}, \ldots, x_{n}^{\gamma}\right)\right)
$$

is true in $\mathfrak{R}$.

Lemma 28 The axiom of replacement holds in $\mathfrak{R}$.

Lemma 29 There is a class $Q$ of the model $\mathfrak{R}$ such that $x \in Q$ if and only if $x$ is a set of conditions compatible with all $p^{(k)}$ in the complete sequence used to define $\mathfrak{N}$.

Lemma 30 There is a class Den of the model $\mathfrak{R}$ such that $\langle y x\rangle \in \operatorname{Den}$ if and only if $x$ is a constant term and $y$ is the set denoted by it. (I.e., Den is the class of pairs $\langle y x\rangle$ such that for some ranked term $u$ denoting $y, x$ is the set ' $u$ ' of Lemma 1.) 
Lemma 31 The power set axiom holds in the model $\Re$.

Lemma 32 The ordinal numbers of $\Re$ are precisely the sets denoted by the constants $\alpha$.

Corollary 32.1 The ordinals of $\mathfrak{N}$ are precisely the ordinals of $\mathfrak{M}$; in particular, if $\mathfrak{M}$ is a well-founded model, then so is $\Re$.

Corollary 32.2 The sets of $\mathfrak{M}$ are well-ordered by a class in the model $\mathfrak{R}$.

Lemma 33 The class form of $\mathrm{AC}$ holds in $\mathfrak{\Re}$.

By these past lemmas, we see that $\mathfrak{R}$ satisfies the axioms of $\Sigma *$. We will now show that $\mathfrak{R}$ satisfies $\boldsymbol{P}\left(\omega_{\zeta}\right) \subset L$.

Proof: We will apply Lemma I.5.4 (of this dissertation), which can be proved in this method of forcing, for the case where $\lambda=\omega_{\zeta}$. Let $\left\{q_{\mu}\right\}_{\mu<\aleph_{\zeta}}$ be a sequence of conditions such that:

(1) $\gamma<\delta \rightarrow q_{\gamma} \subset q_{\delta}$.

We now let

(2) $q=\bigcup_{\mu<\aleph_{\zeta}} q_{\mu}$.

By (1), $q$ is an upper bound for the $q_{\mu}$. We will be done if we can show that $q$ is a set of conditions. We must, therefore, show that $q$ satisfies the conditions of Definition 4. Since each $q_{\mu}$ is a set of conditions, we have

(3) $q_{\mu}=\bigcup_{\alpha \in \operatorname{Reg}_{\zeta}} q_{\mu}^{\alpha}$.

Therefore, by (2) and (3),

(4) $q=\bigcup_{\mu<\aleph_{\zeta}} \bigcup_{\alpha \in \operatorname{Reg}_{\zeta}} q_{\mu}^{\alpha}$.

By the generalized commutativity and associativity of unions, we obtain

(5) $q=\bigcup_{\alpha \in \operatorname{Reg}_{\zeta}} \bigcup_{\mu<\aleph_{\zeta}} q_{\mu \cdot}^{\alpha}$

If we now let

(6) $q^{\alpha}=\bigcup_{\mu<\aleph_{\zeta}} q_{\mu}^{\alpha}$

we obtain

(7) $q=\bigcup_{\alpha \in \operatorname{Reg}_{\zeta}} q^{\alpha}$

where we now need only show that the $q^{\alpha}$, as defined in (6), are of the proper form. But, by (6), any quadruple in $q^{\alpha}$ must be in some $q_{\mu}^{\alpha}$, and hence must be in the form required by Definition 4. We have now verified (i) and (ii) of Definition 4. We must now show that

(8) $\bigcup_{\beta \leqq \alpha} q^{\beta}$ is of cardinality $<\aleph_{\alpha}$, for $\alpha \in \operatorname{Reg}_{\zeta}, \beta \in \operatorname{Reg}_{\zeta}$. 
By (6), we have

(9) $\bigcup_{\beta \leqq \alpha} q^{\beta}=\bigcup_{\beta \leqq \alpha} \bigcup_{\mu<\aleph_{\zeta}} q_{\mu}^{\beta}$.

By reversing the order of unions, we have:

(10) $\bigcup_{\beta \leqq \alpha} q^{\beta}=\bigcup_{\mu<\aleph_{\zeta}} \bigcup_{\beta \leqq \alpha} q_{\mu}^{\beta}$.

But, by (3), we have

(11) $\bigcup_{\beta \leqq \alpha} q_{\mu}^{\beta} \subset \bigcup_{\beta \in \operatorname{Reg}_{\zeta}} q_{\mu}^{\beta}=q_{\mu}$.

Since $q_{\mu}$ is a set of conditions,

(12) $\overline{\overline{\bigcup_{\beta \leqq \alpha} q_{\mu}^{\beta}}}<\aleph_{\alpha}$.

Therefore, since $\aleph_{\zeta}<\aleph_{\alpha}$, and $\aleph_{\alpha}$.is regular, and by (12), we have

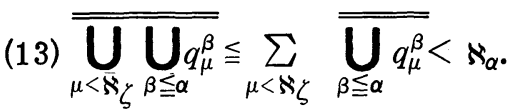

Thus, $q$ satisfies part (iii) of Definition 4. Part (iv) is clear by (1) and (2). Therefore $q$ is a set of conditions and we have satisfied the hypotheses of Lemma I.5.4. Thus we have $\boldsymbol{P}\left(\omega_{\zeta}\right) \subset L$ in $\mathfrak{R}$.

Lemma 34 If $\aleph_{\beta}$ is of cofinality greater than $\aleph_{\alpha}$ in the model $\mathfrak{M}$, then the same holds in the extension $\mathfrak{R}$.

Corollary 34.1 Cardinals are absolute in the extension from $\mathfrak{M}$ to $\mathfrak{R}$.

We must make the same changes in the proof of Lemma 35 as we have made earlier.

Lemma 35 Let $u$ denote a subset of $\aleph_{\alpha}$ in $\mathfrak{n}$. Then there is some $p^{(k)}$ in the complete sequence used to define $\mathfrak{N}$ and some set $\Pi$ of sets of conditions such that:

(i) $p \epsilon \Pi \rightarrow p \subset \Gamma_{\alpha}$.

(ii) $\overline{\bar{\Pi}} \leqq \aleph_{\alpha}$.

(iii) $p^{(k)} \Vdash u \subset \aleph_{\alpha}$.

(iv) If $q^{\prime}$ is an extension of $p^{(k)}$ and $\gamma<\aleph_{\alpha}$, there is some $p \in \Pi$ compatible with $q^{\prime}$ such that either $p \cup p^{(k)} \Vdash \gamma \in u$ or $p \cup p^{(k)} \Vdash \sim(\gamma \in u)$.

In [5], Easton makes the convention that, for singular cardinals $\aleph_{\alpha}, \aleph_{G^{\prime} \alpha}$ is the first cardinal greater than or equal to $\aleph_{G^{\prime} \beta}$ for all $\beta<\alpha$ which are not cofinal with $\aleph_{\alpha}$. We extend our definition of $G$ to all ordinals (instead of merely those $>\zeta$ ) by letting $G^{\prime} \alpha=\alpha+1$ for $\alpha \leqq \zeta$. We then have exactly the same result as Easton's Lemma 36.

Lemma $362^{\aleph_{\alpha}}=\aleph_{G^{\prime} \alpha}$ in the model $\mathfrak{R}$. 
Proof: If $\alpha>\zeta$, the proof follows in exactly the same way as Easton's. If $\alpha \leqq \zeta$, we need to show $2^{\aleph_{\alpha}}=\aleph_{\alpha+1}$, but this is easily proved using the methods of our proof of Theorem I. 3 where we can now use AC (since it holds in $\mathfrak{R}$ ).

We have now completed the proof of Theorem II.7. The following is a summary of the differences between Easton's Theorem and Theorem II.7.

(1) Easton starts with a model of $\Sigma_{*}+\mathrm{GCH}$, while we used a model of $\Sigma_{*}+\mathbf{G C H}+\boldsymbol{p}\left(\omega_{\zeta}\right) \subset \mathrm{L}$.

(2) Easton showed that the power sets of regular cardinals could have any cardinalities compatible with König's theorem. We showed that we could have $\boldsymbol{p}\left(\omega_{\zeta}\right) \subset \mathrm{L}$ and still allow the power sets of cardinals $>\boldsymbol{s}_{\zeta}$ to have any cardinalities compatible with König's theorem.

Thus we see that the assumption of $\boldsymbol{p}\left(\omega_{\zeta}\right) \subset \mathrm{L}$ does not affect the possible cardinalities of regular cardinals greater than $\aleph_{\zeta}$.

In this chapter we have seen the effect that the assumption of $\boldsymbol{P}\left(\omega_{\alpha}\right) \subset \mathrm{L}$ has on $V=L, G C H$, and $A C$. In the next chapter we will study the effect of assuming that all sets of cardinals are constructible.

\section{CHAPTER III}

\section{CONSTRUCTIBILITY WITHOUT REGULARITY}

In the first two chapters we studied the propositions $\boldsymbol{P}(\alpha) \subset L$ for given ordinal numbers $\alpha$. We also mentioned a result of Lévy that says that if every set of ordinal numbers is constructible, and if we assume the axiom of regularity in our set theory, then every set is constructible; i.e., $V=L$. So, in some ways, the presence of the axiom of regularity makes $V=L$ a "least upper bound" for the $\boldsymbol{P}(\alpha) \subset \mathrm{L}$. It then seems natural to ask whether it is necessary to have the axiom of regularity in order to have this situation. We answer this in the affirmative. In fact, we will even go further than this. We will show that if the axiom of regularity does not hold, then it is possible to have a model in which every set that consists solely of constructible elements will itself be constructible (yet in which we do not have $V=L$ ). Thus, we will have a proper decomposition (in ZF without regularity) of $V=L$ into the axiom of regularity and the proposition $\forall x(x \in \vee \wedge x \subset \mathrm{L} \rightarrow x \in \mathrm{L})$. We will formalize these ideas with the following theorems.

The first theorem that we present is weaker than the above-mentioned result of Lévy, but we include it because it gives a good idea of the role that the axiom of regularity plays in relation to $V=L$. Before giving this theorem, we first mention that if the axiom of regularity is true, then it is impossible to have an infinite descending $\epsilon$-chain; i.e., there do not exist sets $x, x_{1}, x_{2}, \ldots$ such that ... $x_{n+1} \epsilon x_{n} \epsilon \ldots \epsilon x_{1} \epsilon x$. (For a proof of this see Rubin [15], p. 117.) In this chapter we shall use $Z F^{*}$ for $Z F$ without the axiom of regularity. 
Theorem III.1 In ZF*, V $=\mathrm{L} \leftrightarrow$

(i) Axiom of regularity

and

(ii) $\forall x(x \in \mathrm{V} . \wedge . x \subset \mathrm{L} \rightarrow x \in \mathrm{L})$.

Proof: If $\mathrm{V}=\mathrm{L}$, then clearly (ii) is true (because the consequence of the implication is true). It is likewise easy to show that $V=L \rightarrow(i)$. (See [15], p. 360, Theorem 14.2.17.) Therefore we have $V=L \rightarrow(i)+(i i)$.

(1) Suppose we have (i) and (ii),

and also suppose it is false that $V=L$; i.e., there is some $x \in V$ such that

(2) $\sim(x \in \mathrm{L})$.

We first note that, since $\phi \epsilon L$, we have

(3) $x \neq \phi$.

By (ii) and (2), there must be some $x_{1}$ such that

(4) $x_{1} \in X$

and yet

(5) $\sim\left(x_{1} \in \mathrm{L}\right)$.

By (5), we see that

(6) $x_{1} \neq \phi$.

Again, by (ii) and (5), there must be some $x_{2}$ such that

(7) $x_{2} \in x_{1}$

(8) $\sim\left(x_{2} \in L\right)$.

This process can be continued for every integer $n$; hence, we will obtain $x, x_{1}, x_{2}, \ldots$ such that, by (4), (7), .. we will have

(9) $\ldots x_{n+1} \in x_{n} \epsilon \ldots \in x_{1} \in X$

which contradicts (i). Thus the theorem has been proved.

We now wish to show that this is a proper decomposition of $V=L$. We will do this by showing that the axiom of regularity does not imply $\forall x(x \in \mathrm{V} . \wedge . x \subset \mathrm{L} \rightarrow x \in \mathrm{L})$ nor does the reverse implication hold. We treat the easy case first:

Theorem III.2 In the field of $\mathrm{ZF}^{*}$, the axiom of regularity does not imply $\forall x(x \in \vee \wedge x \subset \mathrm{L} \rightarrow x \in \mathrm{L})$.

Proof: Suppose it did. Then, by Theorem $\Pi I .1, V=L \leftrightarrow$ the axiom of regularity. But Cohen [3] has exhibited a model in which ZF* and the axiom of regularity hold but in which $\sim(V=L)$.

Theorem III.3 In the field of $\mathrm{ZF}^{*}, \forall x(x \in \mathrm{V} \wedge x \subset \mathrm{L} \rightarrow x \in \mathrm{L})$ does not imply the axiom of regularity. 
Proof: It is enough to find a model $\mathfrak{N}$ in which $\forall x(x \in \vee \wedge x \subset \mathrm{L} \rightarrow x \in \mathrm{L})$ but in which the axiom of regularity fails. We will now proceed to do this. Since we are working with $\mathrm{ZF}^{*}$, we would like to define the subclass of sets which satisfy the axiom of regularity. We do this in a way similar to that which Shoenfield suggests in [20], p. 315 , \$2. He calls a set $x$ regular if each of its subsets $z$ has a minimal element (i.e., $\exists y(y \epsilon z \wedge y \cap z=\phi)$ ). Instead of Shoenfield's notion of regular set, we shall use the related notion of grounded set, whose definition is due to Mendelson [12]. The reason for the use of grounded sets will become clear by Lemma III.3.2.

Definition III.3.1 A set $x$ is grounded $\leftrightarrow$ there is no infinite descending $\epsilon$-chain beginning with $x$. (I.e., there do not exist $x_{1}, x_{2}, \ldots$ such that $\ldots \in x_{n+1} \in x_{n} \epsilon \ldots \in x_{1} \in x_{\text {.) }}$

Lemma III.3.2 $x$ is grounded if and only if every element of $x$ is grounded. Proof:

(1) Suppose $x$ is grounded;

suppose also that we have

(2) $y \in x$

and

(3) $y$ is not grounded.

By (3) and Definition III.3.1, we have $y_{1}, y_{2}, \ldots$ such that

(4) $\ldots \in y_{n+1} \in y_{n} \in \ldots \in y_{2} \in y_{1} \in y_{\text {. }}$

But then, by (2) and (4), we have

(5) $\ldots \in y_{n+1} \in y_{n} \epsilon \ldots \in y_{1} \in y \in x$.

Hence we have

(6) $x$ is not grounded,

which contradicts (1). Now suppose

(7) $x$ is not grounded.

Then there are $x_{1}, x_{2}, \ldots$ such that

(8) $\ldots \in x_{n+1} \in x_{n} \in \ldots \in x_{2} \in x_{1} \in X$.

But then we also have

(9) $\ldots \in x_{n+1} \in x_{n} \epsilon \ldots \in X_{2} \in X_{1}$

and so,

(10) $x_{1}$ is not grounded.

But $x_{1} \in x$; therefore, by (10), there is some element of $x$ that is not grounded. 
We now define our constructible sets in $Z^{*}$. We use the definition that Cohen [3] gives and note that we can use this in $\mathbf{Z F}^{*}$ as well as in $\mathbf{Z F}$ since this definition depends only on the available ordinal numbers, and these are the same in the two systems. To see that the ordinal numbers are the same in $\mathbf{Z F}$ and $\mathbf{Z F}^{*}$, note that the definition of ordinal numbers in $\mathbf{Z} \mathbf{F}^{*}$ (e.g., Rubin [15], p. 176) requires that an ordinal number be well-ordered by the $\epsilon$-relationship. But being well-ordered by $\epsilon$ requires that there not be any infinite descending $\epsilon$-chain; hence, the ordinal number must be grounded.

Definition III.3.3 Let $x$ be a set. The set $x^{\prime}$ is defined as the union of $x$ and the set of all sets $y$ for which there is a formula $\mathfrak{U}\left(z, t_{1}, \ldots, t_{k}\right)$ in $\mathbf{Z F}^{*}$ such that if $\mathfrak{A}_{x}$ denotes $\mathfrak{U}$ with all bound variables restricted to $x$, then for some $\bar{t}_{i}$ in $x, y=\left\{z \in x \mid \mathfrak{A}_{x}\left(z, \bar{t}_{1}, \ldots, \bar{t}_{k}\right)\right\}$.

Definition III.3.4 For $\alpha$ an ordinal number, define $M_{\alpha}$ by:

$$
\begin{aligned}
& M_{0}=\phi \\
& M_{\alpha}=\left(\bigcup_{\beta<\alpha} M_{\beta}\right)^{\prime} .
\end{aligned}
$$

Definition III.3.5 A set $x$ is constructible $(x \in \mathrm{L})$ if $\exists \alpha$ such that $x \in M_{\alpha}$.

Now we introduce into our language a countable number of $\mathrm{O}$-ary function symbols (constants) $x_{1}, x_{2}, \ldots$ and a binary predicate symbol " $\epsilon$ " such that:

$$
\mathrm{x}_{i} \text { " } \epsilon \text { " } \mathrm{x}_{j} \leftrightarrow i=j+1 .
$$

Definition III.3.6 For $\alpha$ an ordinal number, define $\mathrm{N}_{\alpha}$ by:

$$
\begin{aligned}
& N_{0}=\left\{x_{1}, x_{2}, \ldots\right\} \\
& N_{\alpha}=\left(\bigcup_{\beta<\alpha} N_{\beta}\right)^{\prime} .
\end{aligned}
$$

Definition III.3.7 $x \in \Re \leftrightarrow \exists \alpha$ such that $x \in \mathrm{N}_{\alpha}$.

Definition III.3.8 We define $v \in u$ in $\mathfrak{R}$ by:

If $u$ is a set, then $v \in u$ if and only if $v$ is a member of $u$.

If $u=\mathrm{x}_{i}$, then $v \epsilon u$ if and only if $v=\mathrm{x}_{i+1}$.

In other words, if $u=x_{i}$, then $v \epsilon u \leftrightarrow v$ " $\epsilon$ "' $u$. (See Cohen [3] p. 72 for a similar introduction of symbols.)

Lemma III.3.9 If $x \in M_{\alpha}$ and $y \in x$, then there is some $\beta<\alpha$ such that $y \in M_{\beta}$.

Proof: This is evident from Definition III.3.3, Definition III.3.4, and an application of transfinite induction.

Lemma III.3.10 For every $x, y$, and $\alpha$ :

(i) If $x \in \mathrm{N}_{0}$ and $y \in x$, then $y \in \mathrm{N}_{0}$.

(ii) If $x \in \mathrm{N}_{\alpha}$, for $\alpha>0$, and $y \in x$, then there is some $\beta>\alpha$ such that $y \in \mathrm{N}_{\beta}$.

Proof: (i) is clear from Definitions III.3.6 and III.3.8. (ii) is clear from Definitions III.3.3, III.3.6, III.3.8, and an application of transfinite induction. 
Lemma III.3.11 The axioms of $\mathrm{ZF}^{*}$ hold in $\mathrm{L}$.

Proof: In [8], p. 6, Gödel indicates that the axiom of regularity is unnecessary in his construction of L; i.e., even if he does not assume the axiom of regularity in the original theory, he would still get a model $L$ which has all the same properties as when the axiom of regularity is assumed. Then, in [18], Shepherdson proves that the axiom of regularity does not have to be assumed. We proceed to give an informal proof. We note that in Cohen's proof that $L$ is a model for $Z F$, he does not use the axiom of regularity except to verify the axiom of regularity in $L$ (which we do not need) and to show that, for every ordinal number $\alpha, \alpha \in M_{\alpha+1}$ (which shows that the axiom of infinity holds in $L$ ). We can avoid his use of the axiom of regularity in verifying the axiom of infinity in the following way. To verify the axiom of infinity in $L$, we need only show that the ordinal number $\omega$ is in $L$. We note that $\phi \in \mathrm{L}$, and if $x \in \mathrm{L}$, then $x \cup\{x\}$ is in $\mathrm{L}$ (since $\{x\} \in \mathrm{L}$ by the axiom of pairing and $x \cup\{x\}$ is in $L$ by the sub-set axiom). Thus every finite ordinal number is in $L$, and $\omega$ is just the union of all these finite ordinals.

Lemma III.3.12 The axioms of $\mathrm{ZF}^{*}$ hold in $\mathfrak{N}$.

Proof: This is handled in exactly the same way as the previous lemma, except for the axiom of extensionality. But this is shown to be satisfied by Definition III.3.8.

Lemma III.3.13 If $\alpha$ is an ordinal number, then $\mathrm{M}_{\alpha} \subset \mathrm{N}_{\alpha}$ and, therefore, $L \subset \mathfrak{R}$.

Proof: It is sufficient to show that for any sets $a$ and $b, a \subset b \rightarrow a^{\prime} \subset b^{\prime}$. If this can be shown then the proof proceeds by simple transfinite induction.

(1) Suppose $a \subset b$.

(2) Suppose that $x \in a^{\prime}$.

By Definition III.3.3, and (2), there is a formula $\mathfrak{A}\left(z, t_{1}, \ldots, t_{k}\right)$ such that

(3) $x=\left\{z \mid z \in a \wedge \exists \bar{t}_{1} \ldots \bar{t}_{k}\left(\bar{t}_{1} \in a \wedge \ldots \wedge \bar{t}_{k} \in a \wedge \mathfrak{A}_{a}\left(z, \bar{t}_{1}, \ldots, \bar{t}_{k}\right)\right)\right\}$.

But, by (1), we then have, from (3), that

(4) $x=\left\{z \mid z \epsilon b \wedge \exists \bar{t}_{1} \ldots \bar{t}_{k}\left(\bar{t}_{1} \epsilon b \wedge \ldots \wedge \bar{t}_{k} \in b \wedge\left[\mathfrak{H}_{a}\left(z, \bar{t}_{1}, \ldots, \bar{t}_{k}\right) \wedge z \epsilon a \wedge \bar{t}_{i} \epsilon a\right]\right)\right\}$.

Now we let $B$ be the formula

(5) $\mathscr{B}\left(z, t_{1}, \ldots, t_{k}\right) \leftrightarrow z \epsilon a \wedge t_{1} \epsilon a \wedge \ldots \wedge t_{k} \in a \wedge\left[\mathfrak{H}_{a}\left(z, t_{1}, \ldots, t_{k}\right)\right]$.

Since $a \subset b$, we know that

(6) $\left(\mathfrak{U}_{a}\right)_{b} \leftrightarrow \mathfrak{U}_{a}$.

Therefore, by (5) and (6), we have

(7) $\mathfrak{B}_{b}\left(z, t_{1}, \ldots, t_{k}\right) \leftrightarrow z \in a \wedge t_{1} \in a \wedge \ldots \wedge t_{k} \in a \wedge \mathfrak{A}_{a}\left(z, t_{1}, \ldots, t_{k}\right)$.

Hence, by (4) and (7), we have

(8) $x=\left\{z \mid z \epsilon b \wedge \exists \bar{t}_{1}, \ldots, \bar{t}_{k}\left(t_{1} \epsilon b \wedge \ldots \wedge \bar{t}_{k} \epsilon b \wedge \mathfrak{B}_{b}\left(z, \bar{t}_{1}, \ldots, \bar{t}_{k}\right)\right)\right\}$. 
Thus, by (8) and Definition III.3.3,

(9) $x \in b^{\prime}$.

We have thus verified that $a \subset b \rightarrow a^{\prime} \subset b^{\prime}$ and our lemma is proved.

We will now give the key lemma which determined our choice of a model $\mathfrak{N}$.

Lemma III.3.14 If $x$ is $a$ set in $\mathfrak{R}$ and $x$ is grounded, then $x \in \mathrm{L}$.

Proof: It is sufficient to show that if $x$ is grounded and $x \in \mathrm{N}_{\alpha}$ then $x \in \mathrm{M}_{\alpha+1}$. We will prove this by transfinite induction. We note that, by Definition III.3.1 and Definition III.3.6,

(1) if $x$ is grounded, then $\sim\left(x \in \mathrm{N}_{0}\right)$.

Thus, if $x$ is grounded and $x \in \mathrm{N}_{\alpha}$, we know that $\alpha>0$. Our induction hypothesis is: for all $\beta<\alpha$, if $x$ is grounded and $x \in N_{\beta}$, then $x \in M_{\beta+1}$. Let us suppose that

(2) $x$ is grounded

and that

(3) $x \in \mathrm{N}_{\alpha}$.

If $x=\phi$, then $x \in M_{1} \subset M_{\alpha+1}$. Suppose $\sim(x=\phi)$. Then, by Lemma III.3.8 and (3),

(4) $y \in x \rightarrow$ there is some $\beta<\alpha$ such that $y \in \mathrm{N}_{\beta}$.

By Lemma III.3.2, $y$ is grounded, thus by (2), (4), and the induction hypothesis, we have

(5) $y \in M_{\beta+1}$.

By (5), we have

(6) $x \subset M_{\alpha}$.

But we know that, by (6),

(7) $x=\left\{z \in M_{\alpha} \mid z \in x\right\}$.

But, by (6),

(8) $z \in X \leftrightarrow(z \in x)_{M_{\alpha}}$.

Thus, by (7) and (8), we have

(9) $x=\left\{z \in M_{\alpha} \mid(z \in x)_{M_{\alpha}}\right\}$.

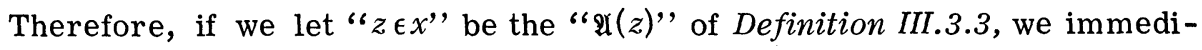
ately know that

(10) $x \in\left(M_{\alpha}\right)^{\prime}$.

But $M_{\alpha}=\bigcup_{\beta \leqq \alpha} M_{\beta}=\bigcup_{\beta<\alpha+1} M_{\beta}$. Therefore, by Definition III.3.4, we have 
(11) $x \epsilon\left(M_{\alpha}\right)^{\prime}=\left(\bigcup_{\beta<\alpha+1} M_{\beta}\right)^{\prime}=M_{\alpha+1}$. This completes the proof of the lemma.

Lemma III.3.15 $x \in \mathrm{L} \rightarrow x$ is grounded.

Proof: Let us suppose that

(1) $x$ is constructible

and that

(2) $x$ is not grounded.

By (2), there are $y_{1}, y_{2}, \ldots$ such that

(3) $\ldots \in y_{n+1} \in y_{n} \epsilon \ldots \in y_{1} \in x$.

By (1), there is an $\alpha$ such that $x \in M_{\alpha}$. Hence by Lemma III.3.9 and (3), we obtain

(4) $\exists \alpha_{1}\left(\alpha_{1}<\alpha \wedge y_{1} \in M_{\alpha_{1}}\right)$.

Likewise we obtain

(5) $\exists \alpha_{2}\left(\alpha_{2}<\alpha_{1} \wedge y_{2} \in M_{\alpha_{2}}\right)$.

In this way we obtain an infinite descending chain of ordinal numbers, which contradicts the well-ordering of $\alpha$ by the $\epsilon$-relationship.

Lemma III.3.16 $\mathfrak{R}$ does not satisfy the axiom of regularity.

Proof: $\mathrm{N}_{0} \in \mathfrak{R}$ and ... $\epsilon x_{n+1} \in x_{n} \in \ldots \in x_{1} \in \mathrm{N}_{0}$.

Lemma III.3.17 In $\mathfrak{N}, \forall x(x \in \mathrm{V}, x \subset \mathrm{L} \rightarrow x \in \mathrm{L})$ holds.

Proof: Let us suppose that

(1) $x$ is a set such that $x \subset \mathrm{L}$.

By Lemma III. 3.15 and (1),

(2) $y \in x \rightarrow y$ is grounded.

By (2) and Lemma III.3.2,

(3) $x$ is grounded.

By (3) and Lemma III. 3.14,

(4) $x \in \mathrm{L}$,

which concludes the proof of the lemma. The proof of Theorem III.3.now follows easily from Lemma III.3.16 and Lemma III.3.17.

The model $\mathfrak{N}$ that we found for Theorem III. 3 is also useful in other applications. It enables us to properly decompose other axioms of set theory.

Corollary III.4 Suppose $\mathrm{B}(x)$ is a predicate on sets such that, in ZF*, B $(\phi)$, $\mathrm{L}(x) \rightarrow \mathrm{B}(x), \mathrm{B}(x) \rightarrow(x$ is grounded $)$ and $\mathrm{V}=\mathrm{B}$ is not provable in ZF. Then the axiom $\mathrm{V}=\mathrm{B}$ can be properly decomposed into

(i) axiom of regularity 
and

(ii) $\forall x(x \in \vee \wedge x \subset \mathrm{B} \rightarrow x \in \mathrm{B})$.

Proof: The proof that $V=B \leftrightarrow(i)+(i i)$ is clear from Theorem III.1 when we note that no property of $L$ was used in that proof other than the fact that $\phi \epsilon \mathrm{L}$. The fact that ( $\mathrm{i}$ ) does not imply (ii) is clear from Theorem III.2 and our hypothesis that $V=B$ is not provable in $Z F$. It now remains to show that (ii) does not imply (i). To show this, we note that in the model $\mathfrak{N}$ of Theorem III.3, by Lemma III.3.14 and Lemma III.3.15.

(1) $x \in \mathrm{L} \leftrightarrow x$ is grounded.

Therefore, by our hypotheses,

(3) $x \in \mathrm{L} \leftrightarrow x \in \mathrm{B} \leftrightarrow x$ is grounded

and the proof proceeds as in Lemma III.3.16 and Lemma III.3.17.

An example of such a predicate $B$ is found in Lévy [9]. He defines a weaker form of $\mathrm{V}=\mathrm{L}$ which he calls $\mathrm{V}=\mathrm{L}_{K}$, where $x \epsilon \mathrm{L}_{K}$ if and only if $x$ is constructible from $K$, where $K$ is a class. He also shows that $\left(\exists K\left(\vee=\mathrm{L}_{K}\right)\right)$ $\leftrightarrow \mathrm{AC}+$ axiom of regularity. Thus, for any $K, \mathrm{~L}_{K}$ satisfies the conditions for Corollary III. 4 .

The proposition

$$
\forall x(x \in \mathrm{V} \wedge x \subset \mathrm{L} \rightarrow x \in \mathrm{L})
$$

is also interesting in itself. Using the methods of Theorem I.3, it can be shown that if we assume this proposition and $A C$, then $G C H$ is provable, even without the axiom of regularity. Thus, even without the use of forcing, we have the following independence theorem:

Theorem III.5 In the field of $\mathrm{ZF}^{*}, \mathbf{G C H}$ does not imply $\mathrm{V}=\mathrm{L}$.

The model $\mathfrak{R}$ of Theorem III. 3 gives us an explicit example of a model of $\mathrm{ZF}^{*}$, in which $\mathrm{GCH}$ holds but not $\mathrm{V}=\mathrm{L}$, since,

Theorem III.6 In $\mathfrak{N}, \mathrm{AC}$ holds.

Proof: We first note a theorem of Cohen [3], p. 95 (which he proves without the axiom of regularity):

Theorem (Cohen) There is a formula $\mathfrak{A}(u, v, X, Y)$, such that, if $Y$ is $a$ well-ordering of the set $X$, the relation $u<v \leftrightarrow \mathfrak{H}(u, v, X, Y)$ induces $a$ well-ordering of the set $X$ !

Thus, in $\mathfrak{N}$, we let $\phi(x)$ be the least $\alpha$ such that $x \in \mathrm{N}_{\alpha}$. Define $x<y$ if $\phi(x)<$ $\phi(y)$ or if $\phi(x)=\phi(y)=\alpha$ and $x$ precedes $y$ in the well-ordering of $N_{\alpha}$. Thus we have a well-ordering of all sets; hence, every set is well-ordered. Thus we have $A C$, and Theorem III. 6 has been proved.

Although $A C$ is a theorem in the model $\mathfrak{N}$ that we constructed above, it is not true that $A C$ follows from $\forall x(x \in \vee \wedge x \subset \mathrm{L} \rightarrow x \in \mathrm{L})$. In fact, we have:

Theorem III.7 In the field of $\mathbf{Z F}^{*}, \mathbf{A C}$ is not a consequence of $\forall x(x \in \vee \wedge x$ $\subset \mathrm{L} \rightarrow x \in \mathrm{L})$. 
Proof: We first note that, by [3], if $\mathbf{Z F}^{*}$ is consistent, then so is $\mathbf{Z F}^{*}+$ $(\sim A C)$. Since $A C$ is equivalent to the well-ordering theorem (Zermelo's Theorem), we can assume that there is a model $\mathfrak{R}$ of $\mathbf{Z F}^{*}$ which contains a set that cannot be well-ordered. Call this set $b$. Now define $M_{\alpha}$ and $L$ as in Definitions III.3.4 and III.3.5. As before, we introduce some new symbols into our language. For each $y \epsilon b$, introduce the constant symbol $x_{y}$.

Definition III.7.1. For $\alpha$ an ordinal number, define $\mathrm{N}_{\alpha}$ by:

$$
\begin{aligned}
& \mathrm{N}_{0}=\left\{x_{y} \mid y \in b\right\} \\
& \mathrm{N}_{\alpha}=\left(\bigcup_{\beta<\alpha} \mathrm{N}_{\beta}\right)^{\prime} .
\end{aligned}
$$

Definition III.7.2. $x \in \mathfrak{R} \leftrightarrow \exists \alpha$ such that $x \in \mathrm{N}_{\alpha}$.

Definition III.7.3. We define $u \in \mathcal{V}$ in $\mathfrak{R}$ by:

(i) If $v$ is a set, then $u \epsilon v$ if and only if $u$ is a member of $v$.

(ii) If $v=\mathrm{x}_{y}$, then $u \epsilon v$ if and only if $u=v$.

The proof that $\mathfrak{N}$ is a model of $\mathbf{Z F}^{*}$ and that $\forall x(x \in \vee \wedge x \subset \mathrm{L} \rightarrow x \in \mathrm{L})$ holds in $\mathfrak{N}$ proceed as in Theorem III.3. We will now show that AC does not hold in $\Re$.

Lemma III.7.4. No cannot be well-ordered.

Proof: Suppose $\mathrm{N}_{0}$ is well-ordered by some relation $<$. We then define the ordering, in $\Re, y<_{\mathfrak{R}} z$ if and only if $x_{y}<x_{z}$ in $\mathfrak{R}$. This induced ordering, $<_{\mathfrak{R}}$, thus well-orders the set $b$ in $\Re$. But this contradicts our assumptions on $b$, and the lemma is proved. This completes the proof of Theorem III.7.

By Theorems III.5, III.6, and III.7, we see the unusual role that the proposition

$$
\forall x(x \in \mathrm{V} \wedge x \subset \mathrm{L} \rightarrow x \in \mathrm{L})
$$

plays with respect to the other strong axioms of set theory. By itself (i.e., without the axiom of regularity) it is not even sufficient for the proof of $A C$. If we assume that both it and $A C$ hold, we then have $G C H$ but not $V=L$. Finally, and perhaps most importantly, together with the axiom of regularity it provides a proper decomposition of $V=L$. Thus, it gives a better explanation of the place of the axiom of regularity in the theory of constructible sets.

We shall investigate some other properties of the proposition $\forall x(x \in \vee \wedge x \subset \mathrm{L} \rightarrow x \in \mathrm{L})$ in a future paper. 


\section{BIBLIOGRA PHY}

[1] Addison, J. W., "Some consequences of the axiom of constructibility," Fundamenta Mathematicae, vol. XLVI (1959), pp. 337-357.

[2] Cohen, P. J., "The independence of the continuum hypothesis," Proceedings of the National Academy of Sciences, vol. 50 (1963), pp. 1143-1148; vol. 51 (1964), pp. 105-110.

[3] Cohen, P. J., Set Theory and the Continuum Hypothesis, New York: Benjamin (1966).

[4] Doss, R., “On Gödel's proof that V = L implies GCH," Notre Dame Journal of Formal Logic, vol. IV (1963), pp. 283-287.

[5] Easton, W. B., Powers of Regular Cardinals, doctoral dissertation, Princeton University (1964).

[6] Gaifman, H., "Self-extending models, measurable cardinals and the constructible universe," mimeographed notes.

[7] Gödel, K., "The consistency of the axiom of choice and the generalized continuum hypothesis," Proceedings of the National Academy of Sciences, vol. 24 (1938), pp. 556-557.

[8] Gödel, K., The Consistency of the Continuum Hypothesis, Princeton: Princeton University Press (1940).

[9] Lévy, A., "A generalization of Gödel's notion of constructibility," The Journal of Symbolic Logic, vol. XXV (1960), pp. 147-155.

[10] Lévy, A., "Definability in axiomatic set theory I," Logic, Methodology and Philosophy of Science, Proceedings of the 1964 Congress (1966), pp. 127-151.

[11] Lévy, A., and R. M. Solovay, "Measurable cardinals and the continuum hypothesis," Israel Journal of Mathematics, vol. 5 (1967), pp. 234-248.

[12] Mendelson, E., "A note on the axioms of restriction and fundierung," Information Sciences, vol. 1 (1968/69), pp. 217-220.

[13] Poss, R. L., "A note on a lemma of J. W. Addison," Notre Dame Journal of Formal Logic, vol. XI (1970), pp. 337-339.

[14] Rowbottom, F., Some Axioms of Infinity Incompatible with the Axiom of Constructibility, doctoral dissertation, University of Wisconsin (1964).

[15] Rubin, J. E., Set Theory for the Mathematician, San Francisco: Holden-Day (1967).

[16] Scarpellini, B., "On a family of models of Zermelo-Fraenkel set theory," Zeitschrift für mathematische Logik und Grundlagen der Mathematik, vol. 12 (1966), pp. 191-204.

[17] Scott, D., "Measurable cardinals and constructible sets," Bulletin de l'Académie Polonaise des Sciences, vol. IX (1961), pp. 521-524.

[18] Shepherdson, J. C., "Inner models for set theory," The Journal of Symbolic Logic, vol. XVI (1951), pp. 161-190. 
[19] Shoenfield, J. R., "On the independence of the axiom of constructibility," American Journal of Mathematics, vol. 81 (1959), pp. 537-540.

[20] Shoenfield, J. R., Mathematical Logic, Reading, Mass.: Addison-Wesley (1967).

[21] Silver, J. H., Some Applications of Model Theory in Set Theory, doctoral dissertation, University of California (1966).

[22] Solovay, R. M., "A nonconstructible $\Delta \frac{1}{3}$ set of integers," Transactions of the American Mathematical Society, vol. 127 (1967), pp. 50-75.

Seminar in Symbolic Logic University of Notre Dame

Notre Dame, Indiana 